\title{
Assessing the Range of Validity of Current Tube Models through Analysis of a Comprehensive Set of Star-Linear 1,4-Polybutadiene Polymer Blends
}

\author{
Ryan Hall, ${ }^{\dagger}$ Priyanka S. Desai, ${ }^{\dagger}$ Beom-Goo Kang, ${ }^{\S, \nabla}$ Qifan Huang, ${ }^{\ddagger}>$ Sanghoon Lee, ${ }^{\circledR \odot}$ \\ Taihyun Chang, ${ }^{\text {ll] David C. Venerus, }}{ }^{\perp, \bigcirc}$ Jimmy Mays, ${ }^{\S}$ Konstantinos Ntetsikas, ${ }^{\#}$
} George Polymeropoulos, ${ }^{\circledR}$ Nikos Hadjichristidis, ${ }^{*}{ }^{\infty}$ and Ronald G. Larson ${ }^{*}$, , ${ }^{\infty}$

\begin{abstract}
${ }^{\dagger}$ Department of Macromolecular Science \& Engineering and ${ }^{\ddagger}$ Department of Chemical Engineering, University of Michigan, Ann Arbor, Michigan 48109, United States

${ }^{\S}$ Department of Chemistry, University of Tennessee, Knoxville, Tennessee 37996, United States

"Department of Chemistry and Division of Advanced Materials Science, Pohang University of Science and Technology (POSTECH), Pohang 37673, Korea

${ }^{\perp}$ Department of Chemical and Biological Engineering and Center for Molecular Study of Condensed Soft Matter, Illinois Institute of Technology, Chicago, Illinois 60616, United States

\#Physical Sciences and Engineering Division, KAUST Catalysis Center, Polymer Synthesis Laboratory, King Abdullah University of Science and Technology (KAUST), Thuwal 23955, Saudi Arabia
\end{abstract}

Supporting Information

ABSTRACT: We blend newly synthesized nearly monodisperse four-arm star 1,4-polybutadienes with various well-entangled linear polymers, confirming the conclusions in Desai et al. [Macromolecules201649 (13)49644977] that advanced tube models, namely, the hierarchical 3.0 and branch-on-branch models [Wang, Z.; et al.J. Rheol.201054 (2)223260], fail to predict the linear rheological data when the pure linear polymers have shorter relaxation times, but within 3-4 orders of magnitude of the star polymer. However, when the linear polymer has a longer relaxation time than the star, our new work, surprisingly, finds that nonmonotonic dependence of terminal relaxation behavior on composition is both

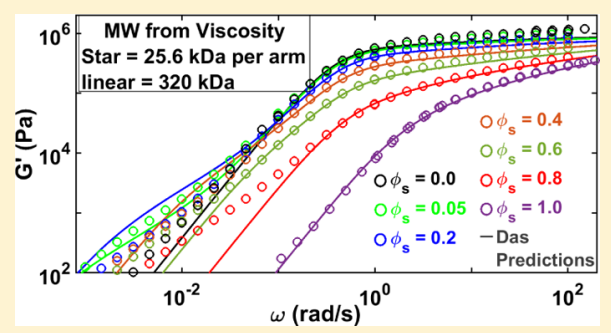
observed experimentally and captured by the models. Combined with previous data from the literature, we present results from over 50 1,4-polybutadiene star-linear blends, suitable for thorough testing of rheological models of entangled polymers.

\section{INTRODUCTION}

The well-known tube model is able to describe nearly quantitatively the relaxation of nearly monodisperse linear polymers using a combination of reptation and contour-length fluctuations. $^{1-7}$ These relaxation processes involve sliding motions, namely, reptation and contour-length fluctuations (CLFs), within a tubelike region defined by the entanglements of an arbitrary chain (i.e., the "probe" chain) with surrounding chains. To describe bidisperse or polydisperse linear polymers, "constraint-release Rouse" (CR-Rouse) dynamics must be added, in which repeated motions of short chains allow the tubes surrounding long chains to migrate. For monodisperse star polymers, constraint release is described by dynamic dilution, a.k.a. "dynamic tube dilation" (DTD), which allows the tube to enlarge its diameter in response to constraint release. Thorough discussions of these mechanisms can be found elsewhere. ${ }^{8-16}$

Using both CR-Rouse relaxation and DTD, Milner et al. ${ }^{17}$ sought to use tube theory to predict the linear rheology of binary star-linear blends. In these blends, the linear component relaxes much faster by reptation than the star arm does by contour-length fluctuations. In the blend of the two, once the linear component relaxes, Milner et al. proposed that the blend undergoes CR-Rouse relaxation, in which the unrelaxed star arm explores a "supertube" whose diameter is defined by star-star entanglements. During this exploration, Milner et al. assumed that contour-length fluctuations of the star arm are unimportant and can be taken to be "frozen" but resume once exploration of the supertube is complete, whereupon the star arm resumes its contour-length fluctuation until it relaxes completely. While this "arm frozen" assumption seemed to yield good agreement between the tube model and the set of experimental data shown in Milner et al., the freezing of the arm fluctuations during CR-Rouse relaxation is artificial. An alternative is to allow the slower species to undergo fluctuations within the original "thin tube", that is, the undilated tube defined by all entanglements of the chain with surrounding chains. The final, "fat tube," option allows the

Received: March 29, 2019

Revised: July 26, 2019 
slower chain to fluctuate in a dilating tube whose diameter is that of the supertube that has been explored up to that time. The generalized tube theory used here does not account for intermediate CR-Rouse physics that spans the difference between the extremes of CR-Rouse fat tube and "thin tube;" however, such physics was explored in the work of Watanabe et al. ${ }^{18-22}$ In a more ideal tube model, the CR-Rouse physics, including both the extreme and intermediate relaxation assumptions, would be implemented self-consistently.

The seeming success of some versions of the tube model to describe linear and star polymers and their blends has inspired the development of generalized tube models to describe the linear rheology of arbitrary blends of linear and branched species. The hierarchical 3.0 model, originally developed by Larson and further refined over the years by Park et al. ${ }^{23}$ and Wang et al. ${ }^{24}$ and the branch-on-branch (or "BoB") model ${ }^{25}$ are two models that extend the work of Milner et al., ${ }^{16,17}$ allowing the prediction of the rheology of mixtures of polymers of varying branched architectures. In addition, the "timemarching algorithm" (TMA) with similar physics, implemented somewhat differently, has been introduced recently by van Ruymbeke and co-workers. ${ }^{26}$ Previous work has shown that these models can, in many cases, predict the rheology of asymmetric polyisoprene stars, ${ }^{23,26} \mathrm{H}$ polymers, ${ }^{23,27}$ pom-pom polymers, ${ }^{27,28}$ comb polymers, ${ }^{23,28,29}$ bidisperse linear $1,4-$ polybutadiene blends, ${ }^{30}$ monodisperse star and linear polymers, ${ }^{26,28}$ and metallocene-catalyzed high-density polyethylene. ${ }^{31}$ However, these "successful" predictions have involved various choices of assumptions, such as the arm frozen, thin tube, and fat tube assumptions for fluctuations during CRRouse relaxation; "disentanglement" mechanisms; and choices of modeling parameters, including the choice of the so-called dilution exponent that determines the relationship between the degree of disentanglement and the diameter of the dilated tube. For star-linear blends, the hierarchical and BoB models, both using the thin tube assumption and no disentanglement, have very similar physics and give quite similar predictions for a given set of parameter values. ${ }^{32}$

To examine tube models and the assumptions within them more rigorously, with less freedom to obtain agreement with data through choice of model assumptions and parameters, Desai et al. ${ }^{32}$ studied a new set of 1,4-polybutadiene starlinear blends consisting of a four-arm star with arm molecular weight (MW) $24 \mathrm{kDa}$ blended with a linear polymer of molecular weight $58 \mathrm{kDa}$, hereby referred to as "24KS" and " $58 \mathrm{KL}$ ", respectively (here, " $\mathrm{K}$ " represents " $\mathrm{kDa}$," while " $\mathrm{S}$ " and "L", respectively, represent "star" and "linear" backbone architecture). Also included in this study are the 42.3KS$105 \mathrm{KL}$ and the $24.5 \mathrm{KS}-7.5 \mathrm{KL}$ 1,4-polybutadiene blend series, respectively, borrowed from Struglinski et al. ${ }^{33}$ and Shivokhin et al. $^{34}$ (We note that Desai et al. modeled the data of Shivokhin et al. using star arm and linear molecular weights of 27.4 and $6.9 \mathrm{kDa}$, respectively, for reasons discussed in their paper.) Since all three of these sets of blends are of nearly identical 1,4-polybutadiene chemistry, the same tube model with the same parameters ought to provide fits to the data for all three sets of data, at the same temperature. Although the hierarchical version of the tube model was in fact able to predict the pure star and linear components, the model was not able to predict, even approximately, the rheology of the 24KS$58 \mathrm{KL}$ blend set, except by invoking the disentanglement mechanism discussed above. But invoking this mechanism led to massive failure to predict the rheology of the $24.5 \mathrm{KS}-7.5 \mathrm{KL}$ blends studied by Shivokhin et al. Thus, no single tube model was able to provide a good fit to all of the (then) available data on star-linear blends of 1,4-polybutadienes. However, in the wake of the hierarchical model's failure, a slip-link model from Schieber and co-workers ${ }^{35-37}$ called the clustered fixed sliplink model (CFSM) was shown by Desai et al. ${ }^{32}$ to be successful in modeling both the $24 \mathrm{KS}-58 \mathrm{KL}$ and the $24.5 \mathrm{KS}-$ $7.5 \mathrm{KL}$ data sets, using a common set of model parameters and no ad hoc adjustments to the model. The success of the CFSM suggests that it captures key physics that the hierarchical model is missing and/or misrepresenting. We briefly note that the CFSM handles the relaxation of entanglements on a probe chain in a more detailed manner than does the tube theory. Thus, the CFSM tends to be computationally slow and limited in the number of polymer chain lengths that can be modeled. Details concerning the development of the CFSM can be found elsewhere. ${ }^{35-39}$

To address the difficulties in the tube model, detailed studies ${ }^{40-44}$ have been conducted to better understand constraint-release physics, in particular dynamic dilution, which is critical for describing the relaxation of branched polymers and polydisperse linear polymers. For branched polymers, deep contour fluctuations (CLFs) of each branch are required for it to achieve terminal relaxation, since, unlike linear polymers, branched polymers are unable to undergo reptation due to the presence of branch points. Thus, the arms of a branched molecule must retract inward toward the branch point, starting from the chain end, to escape entanglements imposed by neighboring chains. Although this deep CLF process is entropically slow, experimental rheological data for pure star-shaped molecules suggest that the arm retraction process is much faster than expected based on CLF alone. ${ }^{45}$ Ball and McLeish ${ }^{15}$ then proposed that, after initial rapid relaxation of the tips of the arms, the entanglements of these arm tips with the unrelaxed portion of a test-chain arm are also relaxed rapidly and thereby accelerate the deep CLF relaxation of the remaining entangled portion of the test-chain arm. This process is called "dynamic dilution" since the rapidly relaxing arm tips act as solvent. A key parameter that controls the rate of dynamic dilution is the dilution exponent, $\alpha$, whose value, thought to be either $\alpha=1$ or $4 / 3$, has been highly debated for over 20 years. ${ }^{16,17,23-26,28,30-33,46-50}$ Different versions of the tube model have used different values of $\alpha$ to fit experimental rheology data.

A recent study by our group ${ }^{44}$ attempted to determine the correct value of the dilution exponent value $(\alpha)$ by measuring the linear rheology of blends of 1,4-polybutadiene four-arm stars mixed with various volume fractions of unentangled linear 1,4-polybutadiene with a molecular weight $1 \mathrm{kDa}$ (" $1 \mathrm{KL}$ "). Unlike most previous studies, this work sought to determine $\alpha$ without relying on a particular tube model, by assuming that the material-dependent parameters controlling the rheology are limited to the plateau modulus $\left(G_{N}^{0}\right)$, the tube-segment frictional Rouse time $\left(\tau_{\mathrm{e}}\right)$, and the number of entanglements per star arm $(Z)$, which are the parameters present in all tube models. Plots of the terminal crossover frequency $\omega_{x, t}$ of the storage and loss moduli, multiplied by the frictional equilibration time $\tau_{\mathrm{e}}$, against the number of entanglements per chain $Z$ for these star-1KL blends collapsed onto corresponding data for 1,4-polybutadiene star melts only for $\alpha=1$ and not for $\alpha=4 / 3$. Other recent studies of Shahid et al., ${ }^{42}$ Huang et al., ${ }^{43}$ and van Ruymbeke and Watanabe ${ }^{40,41}$ also support this finding that $\alpha=1$. 
Our goals for the present study are to further test the tube model and to find more precisely the conditions under which the hierarchical 3.0 model fails. In light of recent work suggesting that the dilution exponent $(\alpha)$ is equal to unity, we specifically test the accuracy of hierarchical model predictions implemented with the "Das" model parameters, which is a commonly used parameter set for 1,4-polybutadienes at $25^{\circ} \mathrm{C}$ in which $\alpha$ is taken to be unity, and we also implement the commonly used thin tube assumption. (A thorough comparison of the predictions of the hierarchical model using thin tube, fat tube, and arm frozen CR-Rouse assumptions against experimental linear rheology data for star-linear 1,4polybutadiene blends was previously presented in Desai et al. $^{32}$ ) In addition, we also provide in this study multiple starlinear blend sets of "benchmark" data that should both inspire improvements in the tube model and allow tests of additional versions of the tube model, as well as other rheological models that are not rooted in tube theory, that might be developed in the future. Thus, to be considered completely successful, a model must predict all of these sets with the same material input parameters, which is an unlikely feat if the model does not accurately capture all of the important physics. This is especially so since data for star-linear blends have proven to be the most difficult to predict using tube models.

For completeness, this paper will report 1,4-polybutadiene star-linear data, referenced at $25{ }^{\circ} \mathrm{C}$, for both newly synthesized and characterized materials and for data already in the literature, which includes the $24 \mathrm{KS}-58 \mathrm{KL}, 24.5 \mathrm{KS}-$ $7.5 \mathrm{KL}$, and $42.3 \mathrm{KS}-105 \mathrm{KL}$ data sets referred to above from Desai et al., ${ }^{32}$ Shivokhin et al., ${ }^{34}$ and Struglinski et al. ${ }^{33}$ The new 1,4-polybutadiene blends studied here include the same four-armed $24 \mathrm{kDa}$ star reported in Desai et al. but here mixed with both a $13.3 \mathrm{kDa}$ linear and a $210 \mathrm{kDa}$ linear 1,4polybutadiene. These blends are labeled $24 \mathrm{KS}-13.3 \mathrm{KL}$ and 24KS-210KL, respectively. The other new 1,4-polybutadiene star-linear blends contain four-arm stars of $25.3 \mathrm{kDa}$ per arm (referenced as "25.3KS"), $44 \mathrm{kDa}$ per arm (referenced as “44KS"), and $47 \mathrm{kDa}$ per arm (referenced as “47KS"). The synthesis and characterization of all new 1,4-polybutadiene stars $(25.3 \mathrm{KS}, 44 \mathrm{KS}$, and $47 \mathrm{KS})$ are described here. The $25.3 \mathrm{KS}$ star is blended with a $73 \mathrm{kDa}$ linear ("73KL") and a $260 \mathrm{kDa}$ linear ("260KL"), while the 44KS star is blended with a $13.3 \mathrm{kDa}$ linear (" $13.3 \mathrm{KL}$ ") polymer, the same linear polymer that is also blended with the $24 \mathrm{KS}$. The $47 \mathrm{KS}$ star is blended with two different linear 1,4-polybutadienes of molecular weights $73 \mathrm{kDa}(73 \mathrm{KL})$ and $260 \mathrm{kDa}(260 \mathrm{KL})$; these are the same linear polymers that are blended with the $25.3 \mathrm{KS}$ sample. In total, these seven new sets of blends, combined with the three previous sets mentioned above, provide us with 10 separate sets of star-linear blends, each containing three or four blend compositions, providing us almost 60 sets of linear rheology data.

This paper is organized as follows. Section II describes the synthesis, preparation, and characterization of the new 1,4polybutadiene samples, as well as the methods of blending and rheological testing of the samples. In Section III, the theoretical and computational methods based on the hierarchical 3.0 tube model are summarized briefly. The results and discussion are presented in Section IV, including the horizontal shift factors obtained when generating master curves of the experimental data, along with an analysis of the associated zero-shear viscosities of the data sets. The linear rheology master curves are then plotted along with predictions of the hierarchical 3.0 model for comparison. Section V reports the conclusions.

\section{MATERIALS AND EXPERIMENTAL METHODS}

II.I. Materials. Six star and five linear 1,4-polybutadienes are presented here in this study. Four of the star polymers are composed of four arms, while the other two have three arms. The four-armed star 1,4-polybutadiene molecules have arm molecular weights of 24, $25.3,44$, and $47 \mathrm{kDa}$; we will refer to these stars as "24KS," "25.3KS," "44KS," and "47KS," respectively, where $\mathrm{K}$ represents $\mathrm{kDa}$ and $\mathrm{S}$ represents star; the number represents the molecular weight of the arm. The $24 \mathrm{KS}, 25.3 \mathrm{KS}, 44 \mathrm{KS}$, and $47 \mathrm{KS}$ samples were synthesized carefully and characterized by gel permeation chromatography (GPC). In addition to GPC, the $24 \mathrm{KS}$ and $44 \mathrm{KS}$ samples were also subjected to characterization by temperature gradient interaction chromatography (TGIC). The synthesis and characterization of $24 \mathrm{KS}$ are detailed in Desai et al., ${ }^{32}$ while details concerning $25.3 \mathrm{KS}, 42 \mathrm{KS}$, and $47 \mathrm{KS}$ can be found in the next section. Data and characterization for the three-armed stars are given in the published literature. Specifically, rheological data for the three-armed star with arm molecular weight of $25.4 \mathrm{kDa}$ (referenced as "25.4KS" in this paper) were taken from Shivokhin et al., ${ }^{34}$ while data for the other threearmed star with arm molecular weight of $42.3 \mathrm{kDa}$ (referenced as "42.3KS") were taken from Struglinski et al. ${ }^{33}$

Three of the five linear 1,4-polybutadiene samples were purchased from Polymer Source. Two of these have molecular weights (as reported by the manufacturer) of $58 \mathrm{kDa}$ [polydispersity index (PDI) $=1.03$, referenced as $58 \mathrm{KL}$, where $\mathrm{L}$ represents linear] and $210 \mathrm{kDa}$ $(\mathrm{PDI}=1.052$, referenced as $210 \mathrm{KL})$. As reported by Polymer Source, the composition of the $58 \mathrm{kDa}$ sample consists of $68 \% 1,4-\mathrm{cis}, 27 \%$ 1,4-trans; and 5\% 1,2-vinyl, and we assume that the composition of the $210 \mathrm{KL}$ sample is similar. The third linear sample from Polymer Source was initially reported to have a molecular weight of $18.2 \mathrm{kDa}$ $($ PDI $=1.15)$; however, we determined through additional GPC testing that the molecular weight of this linear sample is around 13.3 $\mathrm{kDa}(\mathrm{PDI}=1.016$ and hereby referred to as $13.3 \mathrm{KL})$, and through ${ }^{1} \mathrm{H}$ NMR, we determined the 1,2-vinyl content to be $12.5 \%$. The data for the $100 \mathrm{kDa}$ linear sample (referenced to as $105 \mathrm{KL}$ for consistency with the literature) were taken from Struglinski et al., ${ }^{33}$ while data for the $7.5 \mathrm{kDa}$ sample (referenced as $7.5 \mathrm{KL}$ ) were taken from Shivokhin et al..$^{34}$

The above-listed star and linear 1,4-polybutadienes were combined, either in this study or in previous studies, to make the following starlinear blends: $24.5 \mathrm{KS}-7.5 \mathrm{KL}, 24 \mathrm{KS}-13.3 \mathrm{KL}, 24 \mathrm{KS}-58 \mathrm{KL}, 25.3 \mathrm{KS}-$ $73 \mathrm{KL} * *, 24 \mathrm{KS}-210 \mathrm{KL}, 25.3 \mathrm{KS}-260 \mathrm{KL}, 42.3 \mathrm{KS}-105 \mathrm{KL}, 44 \mathrm{KS}-$ $13.3 \mathrm{KL}, 47 \mathrm{KS}-73 \mathrm{KL}$, and $47 \mathrm{KS}-260 \mathrm{KL}$. The italicized blends in the above list were studied in previous papers, while the other blend series were prepared for the current studies. The $25.3 \mathrm{KS}-73 \mathrm{KL}$ blends, marked with “**” above, were generated, and their rheology measured after the conclusions of the paper was drawn based on the other nine blend series to provide a test of these conclusions, as discussed below. This final blend series includes star volume fractions $\left(\phi_{\mathrm{s}}\right)$ of $1,0.9$, $0.6,0.3,0.1$, and 0 . The $24.5 \mathrm{KS}-7.5 \mathrm{KL}$ blend series were prepared with star volume fractions $\phi_{s}=1,0.5,0.2,0.1,0.02$, and 0 . The $24 \mathrm{KS}-13.3 \mathrm{KL}$ blend series consists of $\phi_{\mathrm{s}}=1,0.8,0.4,0.1$, and 0 . For the $24 \mathrm{KS}-58 \mathrm{KL}$ and the $44 \mathrm{KS}-13.3 \mathrm{KL}$ blends, $\phi_{\mathrm{s}}=1,0.9,0.8,0.6$, $0.4,0.2$, and 0 . For the $25.3 \mathrm{KS}-210 \mathrm{KL}$ blend series, $\phi_{\mathrm{s}}=1,0.8,0.6$, $0.4,0.2,0.1$, and 0 . The $24 \mathrm{KS}-260 \mathrm{KL}$ blend series consists of $\phi_{\mathrm{s}}=1$, $0.8,0.6,0.4,0.2,0.05$, and 0 . For the $42.3 \mathrm{KS}-105 \mathrm{KL}$ blends, $\phi_{\mathrm{s}}=1$, $0.75,0.5,0.3,0.2,0.1$, and 0 . Finally, the $44 \mathrm{KS}-73 \mathrm{KL}$ and the $47 \mathrm{KS}-$ $260 \mathrm{KL}$ blends consist of star volume fractions $\phi_{\mathrm{s}}=1,0.8,0.6,0.4,0.2$, and 0 . Presented in Table 1 are the star-linear blends analyzed in this study.

The star-linear 1,4-polybutadiene blends listed above, except for those from the literature $(24.5 \mathrm{KS}-7.5 \mathrm{KL}, 24 \mathrm{KS}-58 \mathrm{KL}$, and $42.3 \mathrm{KS}-$ $105 \mathrm{KL}$ blends), were freshly prepared by first weighing out the pure star and pure linear components in accordance with the desired blend composition. The star and linear samples were then mixed with dichloromethane solvent (Sigma-Aldrich) and stirred at room 
Table 1. Star-Linear 1,4-Polybutadiene Blend Series, Both Newly Prepared and Borrowed from the Literature, That Are Explored in This Study

\begin{tabular}{cl} 
blend series $^{a}$ & \multicolumn{1}{c}{ source } \\
$24.5 \mathrm{KS}-7.5 \mathrm{KL}$ & Shivokhin et al. ${ }^{34}$ \\
$24 \mathrm{KS}-13.3 \mathrm{KL}$ & new \\
$24 \mathrm{KS}-58 \mathrm{KL}$ & Desai et al. ${ }^{32}$ \\
$25.3 \mathrm{KS}-73 \mathrm{KL}$ & new \\
$24 \mathrm{KS}-210 \mathrm{KL}$ & new \\
$25.3 \mathrm{KS}-260 \mathrm{KL}$ & new \\
$42.3 \mathrm{KS}-105 \mathrm{KL}$ & Struglinski et al. ${ }^{33}$ \\
$44 \mathrm{KS}-13.3 \mathrm{KL}$ & new \\
$47 \mathrm{KS}-73 \mathrm{KL}$ & new \\
$47 \mathrm{KS}-260 \mathrm{KL}$ & new
\end{tabular}

${ }^{a} \mathrm{~K}$ represents $\mathrm{kDa}$, while $\mathrm{S}$ and $\mathrm{L}$, respectively, represent star and linear backbone architectures.

temperature within a fume hood. The resulting blend was left in the fume hood for 1 week to evaporate dichloromethane. The blended sample was then transferred to a vacuum chamber for 2 weeks to completely remove excess solvent. The success of solvent removal was checked through a sniff test and by comparing the weight of the blend with its initial mass before the solvent was added. Once the solvent was completely removed, the blend was stored in a freezer to await rheological testing.

II.II. Synthesis. The newly prepared 1,4-polybutadiene star and linear samples (i.e., $73 \mathrm{KL}, 260 \mathrm{KL}, 25.3 \mathrm{KS}$, $44 \mathrm{KS}$, and $47 \mathrm{KS}$ ) were synthesized by anionic polymerization high vacuum techniques and appropriate chlorosilane chemistry as described in our previous paper. ${ }^{44}$ The synthetic procedures are given in Scheme 1A (linear) and $1 \mathrm{~B}$ (stars). Details of the synthesis and molecular characterization are given in the Supporting Information (SI). The synthesis and characterization of 1,4-polybutadiene stars that were taken from the literature, namely, the $24 \mathrm{KS}, 24.5 \mathrm{KS}$, and $42.3 \mathrm{KS}$, are respectively reported in the works of Desai et al., ${ }^{32}$ Shivokhin et al., ${ }^{34}$ and Struglinski et al. ${ }^{33}$

II.III. Characterization. We report in Tables 2 and 3 the molecular weight, polydispersity, and 1,2-vinyl content of the pure linear and pure star 1,4-polybutadienes presented in this study. Table 2 displays the newly prepared linear and star samples, whereas Table 3 reports the star and linear 1,4-polybutadienes obtained from the literature. The data reported in both tables were obtained through gel permeation chromatography (GPC), temperature gradient interaction chromatography (TGIC), and proton nuclear magnetic resonance ( ${ }^{1} \mathrm{H}$ NMR); details of these data can be found in the Supporting Information. As reported in Section IV.II, we checked the molecular weights of the new materials by comparing their zero-shear viscosities with those of other 1,4-polybutadienes in the literature. ${ }^{48,51-54}$ Also, in Section IV.I, we verify (or estimate in some cases) the 1,2-vinyl content, as reported by ${ }^{1} \mathrm{H} \mathrm{NMR}$, of the pure star and pure linear samples by comparing the horizontal Williams-Landel-Ferry (WLF) shift factors of the linear rheology data with those of 1,4polybutadienes from the literature. ${ }^{34,53-57}$

In Table 2, we report the characterization of the newly synthesized (or purchased) pure star and pure linear 1,4-polybutadiene samples. We note that the GPC characterization by Polymer Source for the $13.3 \mathrm{KL}$ sample yielded a molecular weight of $18 \mathrm{kDa}$; however, we concluded that the molecular weight of the linear sample is instead $13.3 \mathrm{kDa}$ from our own GPC testing. We verified this molecular weight by showing that its zero-shear viscosity $\left(\eta_{\mathrm{o}}\right)$ is in better agreement with the molecular weight dependence of the zero-shear viscosities of other 1,4-polybutadiene linear polymers collected from the literature when we assign it a molecular weight of $13.3 \mathrm{kDa}$, rather than $18.2 \mathrm{kDa}$.

Also indicated in Table 2 are both the arm molecular weights of the freshly synthesized four-arm 1,4-polybutadiene stars (i.e., 25.3KS, $44 \mathrm{KS}$, and $47 \mathrm{KS}$ ) inferred by dividing the total molecular weight by 4 , shown in parentheses, and the corresponding molecular weights of the linear arm precursors that were synthesized prior to the introduction of the branching reaction in Scheme 1A of the previous section. The molecular weight of the linear precursor is usually very close to that inferred by dividing the final star molecular weight by the number of arms (assumed to be 4), although in one case (the 44KS sample), the difference is a factor of 1.2. For simplicity, we label these stars by the molecular weights of their linear precursors (i.e., the $44 \mathrm{KS}$ has a linear precursor molecular weight of $44 \mathrm{kDa}$ ). We also note that only the 44KS sample was subjected to TGIC testing. TGIC is considerably more accurate than GPC in the characterization of branched polymers, since the technique is able to resolve peaks with different numbers of arms per polymer, which is generally not possible with GPC. ${ }^{58-61}$ Although we do not have TGIC information for the $25.3 \mathrm{KS}$ and $47 \mathrm{KS}$ 1,4-polybutadiene star samples, we can check the length of the arms by comparing the zero-shear viscosities, which are obtainable through linear rheological testing, to those of other 1,4polybutadiene stars in the literature. Note that the zero-shear viscosity is highly sensitive to arm length and insensitive to the number of arms, except for a modest $(20 \%)$ difference in viscosity between threeand four-arm stars. ${ }^{62}$ Thus, nominally four-arm stars might have some three-arm impurities that will reduce the average molecular weight per arm and only slightly affect the viscosity. For this reason, using the molecular weight of the precursor arm is likely a better estimate of the arm molecular weight than is the total molecular weight of the star divided by four. Details regarding the zero-shear analysis will be presented in Section IV.II, which confirm that the GPC characterizations of the arms of the $25.3 \mathrm{KS}$ and $47 \mathrm{KS}$ stars are reasonable.

Scheme 1. General Reactions for the Synthesis of (A) Linear and (B) Four-Arm Star 1,4-Polybutadiene

(A)
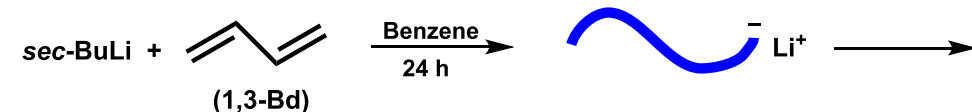

$(1,3-B d)$

(B)
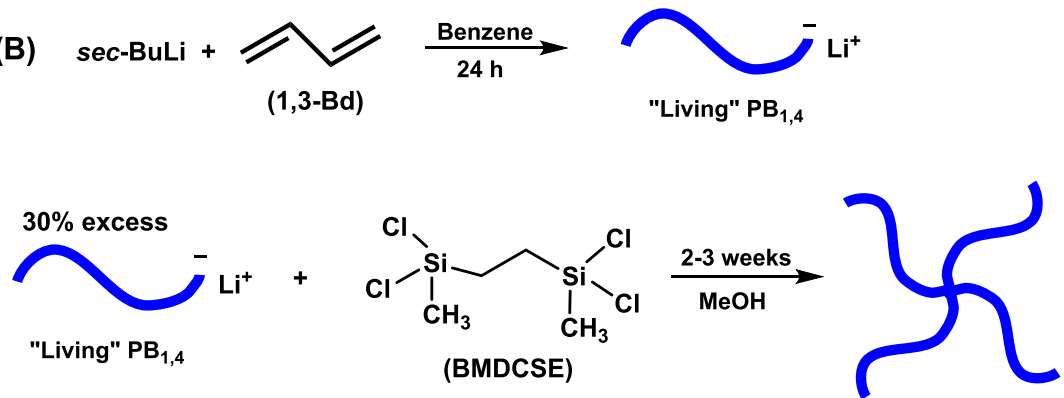
Table 2. Arm Molecular Weight, Polydispersity, and 1,2-Vinyl Content of the Newly Synthesized or Acquired Star and Linear 1,4-Polybutadienes, Obtained through GPC, TGIC, and ${ }^{1} \mathrm{H}$ NMR

\begin{tabular}{|c|c|c|c|c|c|}
\hline sample name & architecture & $M_{\mathrm{w}}^{\mathrm{GPC}}(\mathrm{kDa})^{a}$ & $M_{\mathrm{w}}^{\text {TGIC }}(\mathrm{kDa})^{a}$ & $M_{\mathrm{w}} / M_{\mathrm{n}}^{\mathrm{GPC} b}$ & 1,2-vinyl content $\left({ }^{1} \mathrm{H}\right.$ NMR) (wt \%) \\
\hline $13.3 \mathrm{KL}^{c}$ & linear & 13.3 & & 1.02 & 7 \\
\hline $73 \mathrm{KL}$ & linear & 73 & & 1.04 & 8 \\
\hline $210 \mathrm{KL}^{c}$ & linear & 210 & & 1.05 & \\
\hline $260 \mathrm{KL}$ & linear & 260 & & 1.08 & 7 \\
\hline $25.3 \mathrm{KS}$ & four-arm star (from star) & $25.3(24.6)$ & & $1.03(1.05)$ & 8 \\
\hline $44 \mathrm{KS}$ & four-arm star (from star) & $44(36.5)$ & $44(38.5)$ & 1.07 & \\
\hline $47 \mathrm{KS}$ & four-arm star (from star) & $47(45.8)$ & & $1.05(1.07)$ & 8 \\
\hline
\end{tabular}

${ }^{a}$ In parentheses is the molecular weight per star arm obtained by dividing the molecular weight $\left(M_{\mathrm{w}}\right)$ of the entire star by 4 . Above this is the molecular weight of the linear precursor as determined by GPC using a light scattering detector. ${ }^{b}$ In parentheses is the polydispersity of the star. Above this is the polydispersity of the linear precursor. ${ }^{c}$ Polymer purchased from Polymer Source.

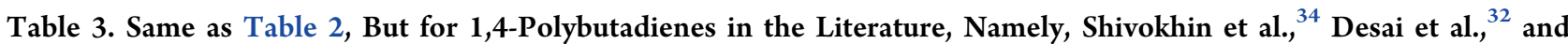
Struglinski et al. ${ }^{33}$

\begin{tabular}{|c|c|c|c|c|c|c|}
\hline sample name & source & architecture & $M_{\mathrm{w}}^{\mathrm{GPC}}(\mathrm{kDa})^{a}$ & $M_{\mathrm{w}}^{\mathrm{TGIC}}(\mathrm{kDa})^{a}$ & $M_{\mathrm{w}} / M_{\mathrm{n}}^{\mathrm{GPC}}$ & 1,2-vinyl content ( ${ }^{1} \mathrm{H}$ NMR) (wt \%) \\
\hline $7.5 \mathrm{KL}$ & Shivokhin et al. & linear & 7.5 & & 1.02 & 10 \\
\hline $24.5 \mathrm{KS}$ & Shivokhin et al. & three-arm star & $24.5(25.3)$ & & 1.05 & 10 \\
\hline $58 \mathrm{KL}$ & Desai et al. & linear & 58 & & 1.04 & 8 \\
\hline $24 \mathrm{KS}$ & Desai et al. & four-arm star & $24(24.3)$ & $24(22.3)$ & 1.05 & \\
\hline $105 \mathrm{KL}$ & Struglinski et al. & linear & 100 & & $<1.1$ & $7^{b}$ \\
\hline $42.3 \mathrm{KS}$ & Struglinski et al. & three-arm star & 42.3 & & $<1.1$ & $10^{b}$ \\
\hline
\end{tabular}

Finally, we note that the maximum polydispersity among all freshly synthesized star and linear 1,4-poybutadiene samples in Table 2 is 1.08 and that this highest polydispersity is for the relatively highmolecular-weight $260 \mathrm{KL}$ melt. The polydispersity of the final stars is somewhat higher than that of the individual arms, which suggests the presence of some dispersity in the number of arms per star in the final product. This supports our decision to use the precursor arm molecular weight rather than final molecular weight to estimate the arm molecular weight of the samples. In addition, the 1,2-vinyl contents of these samples are less than $10 \mathrm{wt} \%$. We also note that the $210 \mathrm{KL}$ and $44 \mathrm{KS}$ samples were not subjected to ${ }^{1} \mathrm{H}$ NMR testing; thus, the 1,2-vinyl contents for these samples are not explicitly known. However, as shown in Section IV.I, we are able to estimate the 1,2vinyl contents of these samples by comparing their horizontal WLF shift factors, obtained from the time-temperature superposition, with those of 1,4-polybutadienes of known 1,2-vinyl content reported in the literature. ${ }^{34,55-57}$

For completeness, we present in Table 3 the characterization results for 1,4-polybutadiene star and linear polymers from the literature ${ }^{32-34}$ that are considered in this study. The samples in Table 3 are nearly monodisperse, and their 1,2-vinyl contents are no more than $10 \mathrm{wt} \%$, which is comparable to those of Table 2 . We note that the 1,2-vinyl content of the $24 \mathrm{KS}$ sample was not reported by Desai et al., ${ }^{32}$ but we are able to estimate it by analyzing the WLF horizontal shift factors of this sample, as described in Section IV.I. We also note that the 1,2 -vinyl contents of the pure $105 \mathrm{KL}$ and $42.3 \mathrm{KS}$ samples were reported from infrared spectroscopy, not ${ }^{1} \mathrm{H}$ NMR. Finally, since TGIC testing was only conducted on the 24KS sample, we validate the GPC molecular weights of the $24.5 \mathrm{KS}$ and $42.3 \mathrm{KS}$ samples by analyzing their zero-shear viscosities, as shown in Section IV.II.

II.IV. Rheology. The linear rheological properties of the newly prepared star-linear blends were measured using $8 \mathrm{~mm}$ parallel plates with a sample gap of $1 \mathrm{~mm}$. As mentioned in Desai et al., 29 the blends were measured on both ARES-LS and RMS-800 rheometers. These tests were performed under strain-control and small-amplitude oscillatory shear flow settings with the same frequency ranges mentioned previously, ${ }^{32}$ at temperatures ranging from 25 to $-100{ }^{\circ} \mathrm{C}$, with the aid of cooling using liquid nitrogen. The resulting linear viscoelastic $G^{\prime}$ and $G^{\prime \prime}$ data were used to generate master curves via time-temperature superposition, at reference temperature $25{ }^{\circ} \mathrm{C}$.
Through these master curves, WLF horizontal shift factors, $a_{T}(T)$, were obtained at each temperature, which are displayed in Section IV.I, for the $13.3 \mathrm{KL}, 73 \mathrm{KL}, 210 \mathrm{KL}, 260 \mathrm{KL}, 24 \mathrm{KS}, 25.3 \mathrm{KS}, 42 \mathrm{KS}$, $44 \mathrm{KS}$, and $47 \mathrm{KS}$ samples and compared to those of 1,4-polybutadiene chemistry found in the literature. ${ }^{5,57}$ In addition to horizontal shifting, the linear viscoelastic data were vertically shifted, $b_{\mathrm{T}}(T)$, in proportion to changes in temperature, as described by Hall et al. ${ }^{44}$ Density changes with temperature were within around $7 \%$ and were usually ignored in generating the master curves. Example master curves both using and ignoring density changes with temperature are shown in Figure S29 in the SI. There is no significant difference between them. (Conclusions from this work rely only on lowfrequency data gathered at $25^{\circ} \mathrm{C}$ where no shifting is required.) The low-temperature data, after time-temperature superposition, reach frequencies high enough to extract the equilibration time, which matches for all samples the "universal" value for 1,4-polybutadiene at $25{ }^{\circ} \mathrm{C}$ given in Park et al., ${ }^{55}$ namely, $3.7 \times 10^{-7} \mathrm{~s}$. As an added measure, for select linear samples, we report rheological data at frequencies that are high enough to show the glassy crossover frequency (which will be shown later in Section IV.I). All unshifted data at each temperature, as well as master curves and shift parameters, will be deposited in the archive https://deepblue.lib. umich.edu/data.

\section{THEORETICAL MODELING}

A recent version of the tube model, the hierarchical 3.0 model, as described in Wang et al., ${ }^{24}$ was used for this study. As discussed in that paper, ${ }^{24}$ for 1,4-polybutadienes at $25^{\circ} \mathrm{C}$, the hierarchical 3.0 model has been implemented with two possible parameter sets: the "Das parameters," which were originally developed for the branch-on-branch (BoB) model, ${ }^{25}$ and the "Park parameters" from the work of Park et al. ${ }^{23} \mathrm{~A}$ fundamental difference between the Park and the Das parameters is the value assigned to the dilution exponent ( $\alpha$ ), which is $\alpha=4 / 3$ and 1 , respectively, for the "Park" and "Das" parameters. The value of $\alpha$ is critical, since it strongly influences the relaxation time of branched polymeric melts, binary blends of branched and linear melts, ${ }^{17,32}$ binary blends 
of linear melts, ${ }^{30}$ and polydisperse linear melts, ${ }^{10-12}$ as discussed in Section I. However, due to recent work conducted by van Ruymbeke and Watanabe, ${ }^{40,41}$ Shahid et al., ${ }^{42}$ Hall et al., ${ }^{44}$ and Huang et al., ${ }^{43}$ there are now strong reasons to believe that $\alpha=1$. Therefore, we will only evaluate here the predictions of the hierarchical model implemented with the Das parameters.

Besides the choice of parameter set, the hierarchical 3.0 model has three options for handling primitive path fluctuations during constraint-release Rouse (CR-Rouse) relaxation. These options, as discussed in the Introduction, are the arm frozen, the thin tube, and the fat tube. In addition to these options for arm fluctuations during CR-Rouse relaxation, there is in the hierarchical model the possibility of allowing a "disentanglement relaxation" to occur when a species (typically a star polymer) has had its entanglement density diluted by constraint release to only $1-3$ remaining diluted entanglements. Please see Wang et al. ${ }^{24}$ for further details regarding these options. In recent work conducted by Desai et al., ${ }^{32}$ the use of both various CR-Rouse assumptions and disentanglement relaxation in predictions yielded mixed results in the modeling of the $24 \mathrm{KS}-58 \mathrm{KL}$ blends; some model predictions had improved agreement with the experimental data, whereas other predictions were worsened. In this paper, we will only utilize the hierarchical model with the Das parameters and with the most commonly used thin tube option, with the acknowledgment that the resulting predictions of experimental data will not yield an allencompassing assessment of tube model accuracy. Disentanglement relaxation will not be considered. We note that these restrictions render the hierarchical model very similar to the BoB model, at least for star-linear blends, and the conclusions drawn here using the hierarchical model also apply when using $\mathrm{BoB}$, as shown in the Supporting Information. We give the Das parameters $G_{N}^{0}, M_{\mathrm{e}}, \tau_{\mathrm{e}}$, and $\alpha$ for 1,4-polybutadiene chemistry in Table 4 below.

Table 4. Das Parameters Used in Hierarchical Model Predictions of 1,4-Polybutadiene

$\begin{array}{ll} & \text { Das model parameters } \\ G_{N}^{0} & 9.7 \times 10^{5} \mathrm{~Pa} \\ M_{\mathrm{e}} & 1836 \mathrm{Da} \\ \tau_{\mathrm{e}} & 2.75 \times 10^{-7} \mathrm{~s} \\ \alpha & 1\end{array}$

\section{RESULTS AND DISCUSSION}

IV.I. Time-Temperature Superposition. We assess and verify the 1,2-vinyl content of the freshly synthesized pure linear (Figure 1) and pure star (Figure 2) 1,4-polybutadiene samples by comparing their WLF horizontal shift factors, plotted against temperature, with those of other 1,4polybutadienes found in the literature, whose 1,2-vinyl content has been reported. The literature polymers shown in Figures 1 and 2 include a $70.6 \mathrm{kDa}$ linear ("70.6KL"), a $24.5 \mathrm{kDa}$ per arm star ("24.5KS"), and a $95.5 \mathrm{kDa}$ linear sample, which were, respectively, taken from Palade et al., ${ }^{57}$ Shivokhin et al., ${ }^{34}$ and Li et al. ${ }^{56}$ These literature WLF horizontal shift factors were reconstructed through use of the time-temperature superposition $C_{1}$ and $C_{2}$ reported in those papers. We report the $C_{1}$ and $C_{2}$ factors of the freshly prepared pure star and pure linear samples from our study in the Supporting Information. In

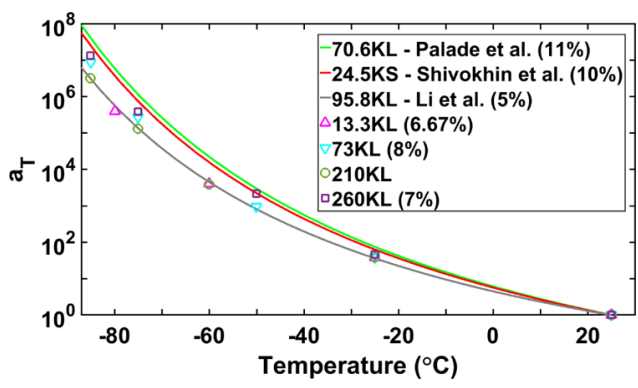

Figure 1. WLF horizontal shift factors for both linear polymers studied here (symbols) and in the literature (lines), which were reconstructed from the reported $C_{1}$ and $C_{2}$ time-temperature superposition constants. In parentheses are the 1,2-vinyl contents reported for each sample, where available. For this plot, and in all subsequent plots, the reference temperature is $25^{\circ} \mathrm{C}$.

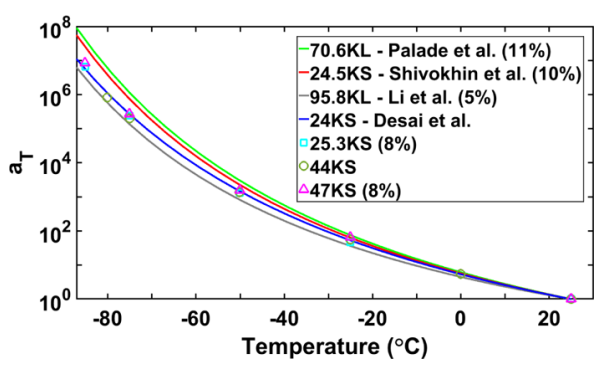

Figure 2. Same as Figure 1, but for freshly prepared four-armed star 1,4-polybutadiene samples. Also included are the WLF shift factors for the pure $24 \mathrm{KS}$ obtained from Desai et al. ${ }^{32}$ plotted here to help estimate its 1,2-vinyl content.

Figure 1, the 1,2-vinyl contents of nearly all freshly synthesized linear 1,4-polybutadine samples of this study (shown as symbols), obtained from ${ }^{1} \mathrm{H} \mathrm{NMR}$, are given in the parentheses of the legend. We verify these and estimate 1,2 contents for samples not so characterized by using benchmarks from the literature (shown as lines in Figure 1). The WLF shift factor curves for the freshly synthesized linear samples (i.e., $13.3 \mathrm{KL}, 73 \mathrm{KL}$, and $260 \mathrm{KL}$ ) are clearly bounded between the $5 \%$ 1,2-vinyl content for the linear polymer reported by $\mathrm{Li}$ et al. ${ }^{56}$ and the $10 \%$ 1,2-vinyl content for the star reported by Shivokhin et al., ${ }^{34}$ which supports the ${ }^{1} \mathrm{H}$ NMR assessment. Since we do not explicitly know the 1,2-vinyl content of the 210KL sample from ${ }^{1} \mathrm{H} \mathrm{NMR}$, we estimate it from its shift factor curve in Figure 1 to be between 5 and 10\%, consistent with the other freshly synthesized linear 1,4-polybutadienes examined in this study.

Similar to Figure 1, we observe in Figure 2 that the freshly synthesized 1,4-polybutadiene stars in this study (symbols) have 1,2-vinyl contents ranging between 5 and $10 \%$, again based on bounds determined by the reference samples from the literature (lines). This observation both helps verify the 1,2-vinyl content determined by ${ }^{1} \mathrm{H}$ NMR for the $25.3 \mathrm{KS}$ and $47 \mathrm{KS}$ samples and provides an estimate of the 1,2-vinyl content of the 44KS sample. In addition, we estimate that the pure $24 \mathrm{KS}$, which was taken from Desai et al., ${ }^{32}$ has a vinyl content between 5 and 10\%. Within this range of 1,2-vinyl contents, our earlier work ${ }^{55}$ shows that the tube model parameters are nearly constant or within experimental error, which is around $25 \%$ for the equilibration time and much less than this for the plateau modulus. 
Corresponding to the horizontal WLF shift factors reported in Figure 1, we present in Figure 3 the resulting $G^{\prime}$ and $G^{\prime \prime}$

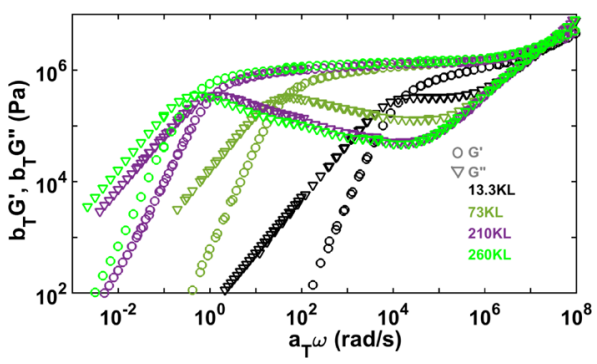

Figure 3. $G^{\prime}$ and $G^{\prime \prime}$ linear rheology master curves of the new linear 1,4-polybutadienes studied here.

linear rheology master curves of the new linear 1,4polybutadiene samples in this study. These data show both the low-frequency crossover and intermediate-frequency crossover of $G^{\prime}$ and $G^{\prime \prime}$ but omit the high-frequency crossover, which is shown for some samples in Figure 4. We note in

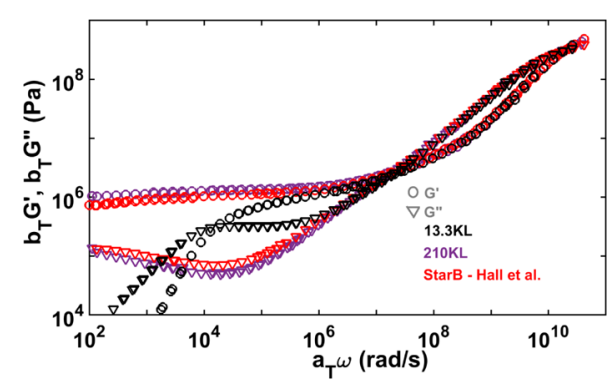

Figure 4. Time-temperature superimposed $G^{\prime}$ and $G^{\prime \prime}$ linear rheology against reduced frequency, plotted up to frequencies in the glassy region, for $13.3 \mathrm{KL}$ and $210 \mathrm{KL}$ linear samples and a fourarm star reported in Hall et al. ${ }^{44}$ that has an arm molecular weight of $65 \mathrm{kDa}$, according to GPC measurement.

Figure 3 that the relaxation curves for all four linear polymers superpose closely at the intermediate $G^{\prime} / G^{\prime \prime}$ crossover region to the right of the rubbery plateau, indicating that they possess similar 1,2-vinyl content and have equilibration time $\tau_{\mathrm{e}}$ consistent with the universal value, $\tau_{\mathrm{e}}=3.7 \times 10^{-7} \mathrm{~s}$, reported in Park et al. ${ }^{55}$ We also note that, except for the sample with lowest molecular weight, $G^{\prime}$ data for these linear samples converge to a similar plateau modulus $\left(G_{N}^{0}\right)$ at frequencies higher than $10^{4} \mathrm{rad} / \mathrm{s}$. The $13.3 \mathrm{KL}$ sample presumably fails to reach a similar value of $G_{N}^{0}$ because of the small number of entanglements in this sample. As noted previously, in addition to horizontal shifting a vertical shift factor $\left(b_{\mathrm{T}}\right)$, proportional to absolute temperature, is used in the generation of rheological master curves. This method used here is not the only vertical shifting approach possible; other methods include (A) omitting vertical shifting $\left(b_{\mathrm{T}}=1\right)^{34}$ or $(\mathrm{B})$ utilizing a statistical shifting approach. ${ }^{32}$

Figure 4 focuses on moderate- and high-frequency regimes of the $13.3 \mathrm{KL}$ and $210 \mathrm{KL}$ linear samples and of the pure StarB four-arm 1,4-polybutadiene star with an $65 \mathrm{kDa}$ molecular weight per arm measured by GPC, as reported in Hall et al. ${ }^{44}$ The very close superposition of the data sets throughout the transition and glassy regions (above a frequency of around $10^{7}$ $\mathrm{rad} / \mathrm{s}$ ) suggests that the molecular weight of the $13.3 \mathrm{KL}$ sample is high enough to avoid a significant change in segmental friction. Thus, no adjustments for changes in friction, either due to small molecular weights or to the 1,2vinyl content, are needed for any of the data reported here.

Similar to Figure 3, we report in Figure 5 the linear rheology of the newly synthesized four-arm 1,4-polybutadiene stars in

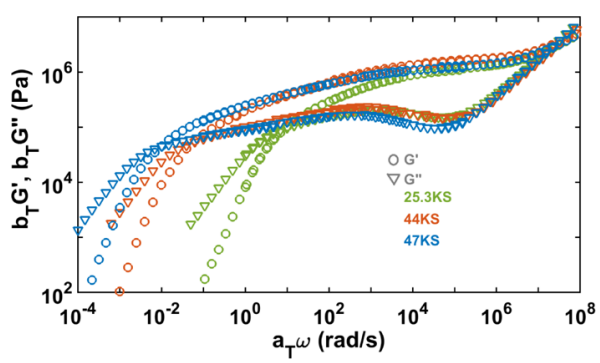

Figure 5. Same as Figure 3 but for the freshly synthesized symmetric four-arm star 1,4-polybutadiene 25.3KS, 44KS, and 47KS melts.

this study. Once again, we observe close superposition of the rheology data sets at the intermediate crossover frequency to the right of what would be the plateau region in a linear sample, signifying a similarity in the 1,2-vinyl content among the samples. We note that the plateau modulus $\left(G_{N}^{0}\right)$ we infer for the 44KS sample is slightly higher than those of the $25.3 \mathrm{KS}$ and $47 \mathrm{KS}$ samples, which is observable in the higher $G^{\prime}$ values for this sample between frequencies of $10^{3}$ and $10^{7} \mathrm{rad} / \mathrm{s}$. This difference in $G_{N}^{0}$ may arise from imperfections in rheological testing at reduced temperatures and/or imperfections of the temperature-dependent vertical shifting, $b_{\mathrm{T}}$, used to generate the rheological master curves. At any rate, this modest difference should have little effect on the main results of this paper.

IV.II. Analysis of Zero-Shear Viscosity. We now assess the accuracy of the GPC and TGIC molecular weight measurements of the 1,4-polybutadienes reported in Tables 2 and 3 by comparing the zero-shear viscosities $\left(\eta_{\mathrm{o}}\right)$ of these polymers, obtained from the linear rheology data, with those of other 1,4-polybutadienes found throughout the literature. In addition, we obtain from these viscosity data estimates of the molecular weights that we will use, along with the molecular weights from GPC, in the hierarchical model, as discussed below. Throughout this paper, the hierarchical model is implemented with the Das parameters and thin-tube CR-Rouse relaxation, as alluded to earlier.

Figure 6 depicts the zero-shear viscosities, scaled by molecular weight to the 3.4 power, of the linear 1,4polybutadienes explored in this study (closed circles) compared with literature sources, which include Colby et al. $^{51}$ (open squares) and Struglinski et al. ${ }^{52}$ (open triangles). We scaled the zero-shear viscosity to reduce the range of the $y$ axis and improve the clarity of deviations among the data depicted. We note that there are two sets of zero-shear viscosity data from Colby et al. for linear polymers with molecular weights below $10000 \mathrm{Da}$. The "Colby et al." data are the unadjusted zero-shear viscosities extracted from linear rheology, whereas the "Colby et al. (free volume)" data were adjusted to correct for the reduction in the segmental friction coefficient that occurs in low-molecular-weight melts. Also included in Figure 6 are a trend line (black line) that fits the reference data of Colby et al. and of Struglinski et al. and a (green) line showing predictions of the hierarchical model, 


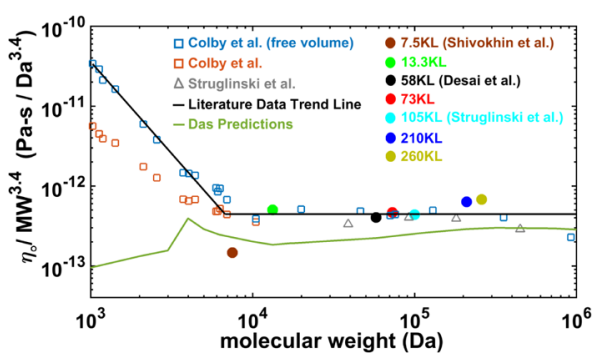

Figure 6. Scaled zero-shear viscosities of individual linear 1,4polybutadienes, from this work and from Struglinski et al., ${ }^{33}$ Desai et al., ${ }^{32}$ and Shivokhin et al. ${ }^{34}$ (solid circles) and from reference sets of multiple samples from the literature ${ }^{51,52}$ (open symbols) versus molecular weight. The zero-shear viscosities are scaled by the molecular weight (MW) to the 3.4 power. Also plotted are zeroshear viscosities predicted by the hierarchical model (green line, labeled "Das predictions") and a power law fit to the literature benchmark data (black line).

which of course fails drastically at low molecular weights where melts are unentangled.

We observe that in Figure 6 the zero-shear viscosities of the linear 1,4-polybutadiene polymers in this study, given by solid symbols, agree reasonably with the black trend line summarizing the literature data, which are given by open symbols. An exception is the 7.5KL melt reported by Shivokhin et $\mathrm{al}^{34}$ whose $\eta_{\mathrm{o}}$ value is roughly a factor of 3 below the black trend line along the $y$ axis, indicating that the molecular weight of this linear sample is possibly lower than the reported value, $7.5 \mathrm{kDa}$. To achieve reasonable superposition with the black trend line, the $7.5 \mathrm{KL}$ melt would instead need to be assigned a molecular weight ranging between 5 and $6 \mathrm{kDa}$. (We noted in the Introduction that Desai et al. ${ }^{32}$ assigned this melt a molecular weight of $6.9 \mathrm{kDa}$.) The zero-shear viscosities of both the $210 \mathrm{KL}$ and the $260 \mathrm{KL}$ melts are somewhat above the black trend line, by a factor of 1.5 for the $210 \mathrm{KL}$ and a factor of 1.6 for the $260 \mathrm{KL}$ melt. To achieve agreement with the trend line, the molecular weight of the $210 \mathrm{KL}$ melt would have to be increased to roughly $235 \mathrm{kDa}$, while that of the $260 \mathrm{KL}$ melt would need to be roughly $295 \mathrm{KDa}$, which are likely within the error of the GPC measurements of these melts. Also, in Figure 6, with the exception of the 7.5KL melt taken from Shivokhin et al., the zero-shear viscosities predicted by the hierarchical model (green line) are notably lower than both the linear melts comprising the star-linear blends of this study (closed circles) and the other linear polymers found in the literature. ${ }^{45,46}$

Figure 7 plots the zero-shear viscosities against the star-arm molecular weight of both the freshly synthesized and literature $^{32-34}$ star 1,4-polybutadiene samples analyzed in this

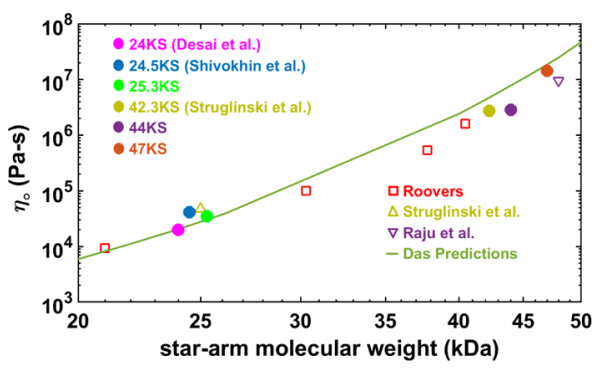

Figure 7. Same as Figure 6, except for star polymers and the viscosities are not rescaled. paper (closed circles) as well as those for other benchmark star 1,4-polybutadiene polymers from the literature. ${ }^{34,43,53}$ Also presented is the plot of the zero-shear viscosities from hierarchical model predictions (green line). The zero-shear viscosities for both the freshly prepared and the literature stars superpose within scatter with the benchmark data represented by open symbols. Therefore, it is reasonable to assume that the arm molecular weights of the freshly prepared $25.3 \mathrm{KS}$, 44KS, and 47KS samples, which were only subjected to GPC analysis, are relatively accurate and that the samples are largely composed of four-arm star molecules. In addition, the zeroshear viscosities predicted by the hierarchical model are roughly equal to the measured values except for the highermolecular-weight values, which are over-predicted by a factor of 2 on average.

The zero-shear viscosities presented in Figures 6 and 7 indicate that the pure star and pure linear 1,4-polybutadienes used in the star-linear blends of this study are reasonably well characterized, although we note that the two high-molecularweight linear polymers used in our study, $210 \mathrm{KL}$ and $260 \mathrm{KL}$, may have a higher molecular weight than indicated by GPC, since their viscosities in Figure 6 lie above the trend line from the literature, and the $7.5 \mathrm{KL}$ sample may have lower molecular weight than the value obtained by GPC characterization. In addition, the hierarchical model with $\alpha=1$ seems to systematically underpredict the viscosity of the pure linear melts and somewhat overpredict the viscosity of the stars with arm molecular weight above around $30 \mathrm{kDa}$. Thus, to correct for modest random errors in characterization and counteract systematic errors in the tube model for the pure materials, we will use in our predictions for the blends both the GPC molecular weights and molecular weights that are modestly adjusted to obtain better agreement with the zero-shear viscosity. These viscosity-adjusted molecular weights are reported in Table 5. The maximum difference between the zero-shear viscosities from model predictions with revised molecular weights and the experimental values is a factor of 1.2. For the star polymer samples, the molecular weight reported is the arm molecular weight. We observe in Table 5 that, as expected, the molecular weight used in the hierarchical model prediction for each linear sample is consistently higher than the associated GPC molecular weight, with the exception of the $7.5 \mathrm{KL}$ sample reported by Shivokhin et al. ${ }^{34}$ The molecular weights of the star polymers used in the model predictions of viscosity in Table 5 are very similar to the molecular weights measured by GPC, for molecular weights below $30 \mathrm{kDa}$, but somewhat lower than the GPC molecular weights for higher arm lengths, as reported in Tables 2 and 3. These results are consistent with earlier observations; when Das parameters, with $\alpha=1$, are used, the hierarchical model is typically not able to fit both star and linear molecules of the same species as well as when $\alpha=1$ is used, ${ }^{24}$ and so the Das parameters (other than $\alpha$ ) provide a compromise that underpredicts the viscosities of the linear polymers but overpredicts those of stars. A better simultaneous prediction of both the pure star and pure linear viscosities is obtained for $\alpha=4 / 3{ }^{24}$ As remarked earlier, however, the choice $\alpha=4 / 3$ is no longer tenable, based on recent studies. Thus, the systematic increase in molecular weight needed to match viscosities of the linear polymers is likely offsetting some systematic error in the hierarchical model, which exists in the Bob model as well. For example, the tube model for branched polymers is based on dynamic tube dilation (DTD) ${ }^{15,16}$ which 
Table 5. Experimental Zero-Shear Viscosities (Column 4) of the Pure Star and Pure Linear 1,4-Polybutadienes in This Study and Viscosities (Column 3) Computed from the Hierarchical Model with the Das Parameters, Using Molecular Weights (Column 2) Chosen to Fit the Experimental Zero-Shear Viscosities ${ }^{a}$

\begin{tabular}{lccc} 
sample & $\begin{array}{c}\text { experimental zero- } \\
\text { shear viscosity } \\
(\mathrm{Pa} \mathrm{s})\end{array}$ & $\begin{array}{c}\text { hierarchical Das } \\
\text { zero-shear viscosity } \\
(\mathrm{Pa} \mathrm{s})\end{array}$ & $\begin{array}{c}\text { hierarchical Das MW } \\
\text { approximations } \\
(\mathrm{kDa})^{b}\end{array}$ \\
\hline $7.5 \mathrm{KL}^{c}$ & 2.19 & 1.98 & 6.25 \\
$13.3 \mathrm{KL}$ & 52.7 & 46.4 & 17 \\
$58 \mathrm{KL}^{d}$ & $6.35 \times 10^{3}$ & $6.56 \times 10^{3}$ & 69 \\
$73 \mathrm{KL}$ & $1.6 \times 10^{4}$ & $1.59 \times 10^{4}$ & 88 \\
$105 \mathrm{KL}^{e}$ & $4.37 \times 10^{4}$ & $4.18 \times 10^{4}$ & 115 \\
$210 \mathrm{KL}$ & $7.91 \times 10^{5}$ & $7.61 \times 10^{5}$ & 260 \\
$260 \mathrm{KL}$ & $1.75 \times 10^{6}$ & $1.56 \times 10^{6}$ & 320 \\
$24 \mathrm{KS}{ }^{d}$ & $2.01 \times 10^{4}$ & $2.04 \times 10^{4}$ & 24 \\
$24.5 \mathrm{KS}{ }^{c}$ & $4.12 \times 10^{4}$ & $3.92 \times 10^{4}$ & 26.2 \\
$25.3 \mathrm{KS}$ & $3.5 \times 10^{4}$ & $3.32 \times 10^{4}$ & 25.6 \\
$42.3 \mathrm{KS}{ }^{e}$ & $2.75 \times 10^{6}$ & $2.4 \times 10^{6}$ & 40 \\
$44 \mathrm{KS}^{2}$ & $2.86 \times 10^{6}$ & $2.78 \times 10^{6}$ & 40.5 \\
$47 \mathrm{KS}^{2}$ & $1.43 \times 10^{7}$ & $1.2 \times 10^{7}$ & 45.5
\end{tabular}

${ }^{a}$ The footnotes identify the data obtained from the literature measured in this work. Any polymer samples listed without notation are newly introduced in this study. ${ }^{b}$ Molecular weight per star arm is reported for the star samples. ${ }^{c}$ Source: Shivokhin et al. ${ }^{34}{ }^{d}$ Source: Desai et al. ${ }^{32}{ }^{e}$ Source: Struglinski et al. ${ }^{33}$

is phenomenological and is unlikely to provide a completely accurate description of branch relaxation. Recent works ${ }^{18,63,64}$ exploring the physics of star polymers, in particular, suggest that both entanglement release near the free ends and entanglement creation near branch points are involved in the dynamics of star arm relaxation and are not properly represented by the DTD theory. In addition to errors in the theory, there are also likely to be errors in the molecular weight characterizations of the samples, which will affect the match of theory to experiment. Thus, to make sure that our conclusions are robust against errors in molecular weight characterization, we will make hierarchical model predictions using star and linear molecular weights from both GPC measurements and from zero-shear viscosity assessments. We note that in the case of the pure stars, the GPC molecular weights used in model predictions correspond with the linear precursor that was characterized prior to the branching reaction. In the main text, we will show summary plots of our results for both choices of molecular weight characterization. To reduce the number of plots in the main text, comparisons of experimental and predicted $G^{\prime}$ curves for select blends will be presented for the GPC and the viscosity-based molecular weights, and the corresponding $G^{\prime \prime}$ curves are given in the SI. These plots show that the conclusions drawn from our work are the same whether we use molecular weights based on viscosity or based on the GPC characterizations of the molecular weights.

IV.III. Evaluation of Hierarchical Model Using StarLinear Blend Rheology Data. As stated in a previous section, we will implement the hierarchical model with the Das parameters set only, which takes the dilution exponent value $(\alpha)$ to be unity and uses the thin tube assumption. In addition, we will not employ the disentanglement relaxation option. The star-linear blends analyzed in this study are divided into two categories. One category consists of star-linear blends in which the star component has an arm molecular weight ranging between 20 and $30 \mathrm{kDa}$; this includes the $24.5 \mathrm{KS}-$ $7.5 \mathrm{KL}$ blends taken from Shivokhin et al., ${ }^{34}$ the $24 \mathrm{KS}-13.3 \mathrm{KL}$ blends, 24KS-58KL blends taken from Desai et al., ${ }^{32} 25.3 \mathrm{KS}-$ $73 \mathrm{KL}$ blends $* *, 24 \mathrm{KS}-210 \mathrm{KL}$ blends, and $25.3 \mathrm{KS}-260 \mathrm{KL}$ blends. (The labeling of one of the blends with asterisks is explained below.) The other category consists of star components with arm molecular weights ranging between roughly $40 \mathrm{kDa}$ and $50 \mathrm{kDa}$; this includes the $42.3 \mathrm{KS}-105 \mathrm{KL}$ blends taken from Struglinski et al., ${ }^{33} 44 \mathrm{KS}-13.3 \mathrm{KL}$ blends, $47 \mathrm{KS}-73 \mathrm{KL}$ blends, and $47 \mathrm{KS}-260 \mathrm{KL}$ blends. To save space, we report in Figures 10-14 only the linear rheology data of star-linear blends with star-arm molecular weights ranging between 20 and $30 \mathrm{kDa}$. The Supporting Information presents the corresponding results for the star-linear blends with stararm molecular weights between 40 and $50 \mathrm{kDa}$, as well as the $25.3 \mathrm{KS}-73 \mathrm{KL}$ blend series. We note that the $25.3 \mathrm{KS}-73 \mathrm{KL}$ blends (marked with ** above) were prepared and tested after the other results had been gathered and conclusions of the paper had been made and written up, and this last data set was gathered to confirm these conclusions.

We begin our assessment of the hierarchical model by plotting in Figure 8 what we will here call the "near-terminal frequency" of the blends $\omega_{n t, b l e n d}$ normalized by the nearterminal frequency of the pure star $\omega_{\text {nt,star, }}$ as a function of the star volume fraction $\left(\phi_{\mathrm{s}}\right)$ from both model predictions (lines) and experimental data (symbols). Both "near-terminal frequencies," $\omega_{\mathrm{nt}, \mathrm{blend}}$ and $\omega_{\mathrm{nt}, \mathrm{star}}$, are defined as the frequency
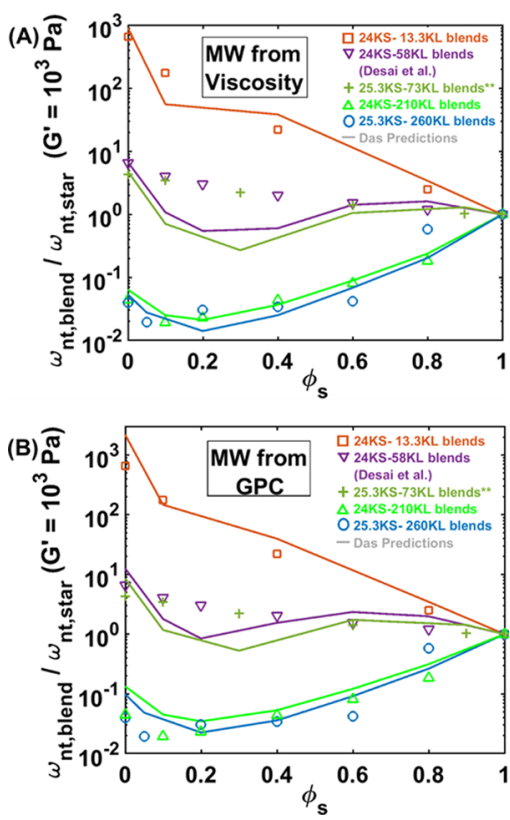

Figure 8. "Near-terminal relaxation frequency" of star-linear blends, defined as the frequency at which $G^{\prime}=10^{3} \mathrm{~Pa}$ and normalized by the near-terminal frequency of the pure star (also the frequency at which $G^{\prime}=10^{3} \mathrm{~Pa}$ ), versus the star volume fraction, $\phi_{\mathrm{s}}$. Symbols are experimental data, and lines are hierarchical model predictions generated with Das parameters and thin tube option as in all subsequent figures. (A) Molecular weights used in model predictions obtained from zero-shear viscosities are taken from Table 5 and (B) molecular weights were measured by GPC. The $24 \mathrm{KS}-58 \mathrm{KL}$ experimental blend data was taken from Desai et al. ${ }^{32}$ We note that the $25.3 \mathrm{KS}-73 \mathrm{KL}$ blend series is marked with $* *$ in the legend because it was prepared and tested after the rest of the samples to confirm the conclusions presented in this work. 
at which $G^{\prime}=10^{3} \mathrm{~Pa}$. We choose this definition of nearterminal frequency because we found that the more obvious choice, namely, the frequency at the terminal crossover of $G^{\prime}$ and $G^{\prime \prime}$, is too high a frequency to capture adequately the predictive failure of the tube model in the terminal region for some of the blends. The frequency at which $G^{\prime}=10^{3} \mathrm{~Pa}$ is low enough to capture this failure, and the modulus $G^{\prime}=10^{3} \mathrm{~Pa}$ is high enough to be largely free from measurement error. We normalize $\omega_{\text {nt,blend }}$ by $\omega_{\text {nt,star }}$ to more easily judge the success or failure of model predictions by the magnitude of the difference in near-terminal relaxation time between the blend and the pure star. Figure 8A displays model predictions that utilize the viscosity-based molecular weights reported in Table 5, whereas the molecular weights from GPC measurements are used in model predictions in Figure $8 \mathrm{~B}$.

We observe in Figure 8A that model predictions superpose rather well with experimental data for most star-linear blend sets. The $24 \mathrm{KS}-13.3 \mathrm{KL}$ blend series, for example, is captured reasonably well by the model, with the exception of the $\phi_{\mathrm{s}}=$ 0.1 blend. However, as observed earlier in the work of Desai et al., ${ }^{32}$ hierarchical model predictions fail when the relaxation time of the pure linear increases to within 3-4 orders of magnitude of the star polymer, which is the case for the 24KS$58 \mathrm{KL}$ and the $25.3 \mathrm{KS}-73 \mathrm{KL}$ blend series. Specifically, the model predicts a non-monotonic dependence of the nearterminal frequency on the star volume fraction, which is not observed in the experimental data. The $24 \mathrm{KS}-210 \mathrm{KL}$ and the $25.3 \mathrm{KS}-260 \mathrm{KL}$ blend series also present predictions of nonmonotonic behavior, and, surprisingly, this non-monotonic behavior is actually observed in these blends. In Figure 8B, we use the GPC molecular weights instead of those determined by fits with the zero-shear viscosities of the pure star and the pure linear polymers; thus, the predictions of the pure linear polymers do not superpose as closely with the data in Figure $8 \mathrm{~B}$ as they due in Figure 8A. However, we observe in Figure 8B the same trend in model prediction successes and failures as we do in Figure 8A. Specifically, in Figure 8B, the model predicts reasonably well the $24 \mathrm{KS}-13.3 \mathrm{KL}$ blends. Model predictions of the $24 \mathrm{KS}-58 \mathrm{KL}$ blend series show a non-monotonic dependence of $\omega_{\text {nt,blend }}$ on blend composition, which is not seen experimentally. Finally, in Figure 8B, non-monotonic model predictions of the $24 \mathrm{KS}-210 \mathrm{KL}$ and the $25.3 \mathrm{KS}-$ $260 \mathrm{KL}$ blends are consistent with those of Figure $8 \mathrm{~A}$. This is the first report of experimentally validated non-monotonic dependence of terminal relaxation on blend composition in the literature, which we will discuss in more detail in what follows.

Similarly to Figure 8, we define in Figure 9 a characteristic near-terminal frequency for each blend $\left(\omega_{\text {nt,blend }}\right)$ as the frequency at which $G^{\prime}$ reaches the value of $1000 \mathrm{~Pa}$; however, in Figure 9, we plot data for the star-linear blends (symbols) for stars with arm molecular weight above $40 \mathrm{kDa}$, namely, the $44 \mathrm{KS}-13.3 \mathrm{KL}, 47 \mathrm{KS}-73 \mathrm{KL}, 47 \mathrm{KS}-260 \mathrm{KL}$, and $42.3 \mathrm{KS}-$ $105 \mathrm{KL}$ blends taken from Struglinski et al. ${ }^{33}$ Each $\omega_{\text {nt,blend }}$ value was again normalized by the terminal frequency of the respective pure star $\left(\omega_{\text {nt,star }}\right)$ of the blend series, also at $G^{\prime}=$ $1000 \mathrm{~Pa}$. Figure 9A displays model predictions that utilize the molecular weights reported in Table 5 , whereas the molecular weights from GPC measurements are used in model predictions in Figure 9B. Shown in Figure 9A, the hierarchical model predictions (lines) are mostly in reasonable agreement with the $44 \mathrm{KS}-13.3 \mathrm{KL}$ and $47 \mathrm{KS}-73 \mathrm{KL}$ star-linear blend data. The largest deviation from the $44 \mathrm{KS}-13.3 \mathrm{KL}$ data is at star volume fractions $\left(\phi_{\mathrm{s}}\right)$ of 0.2 and 0.4 , where the model
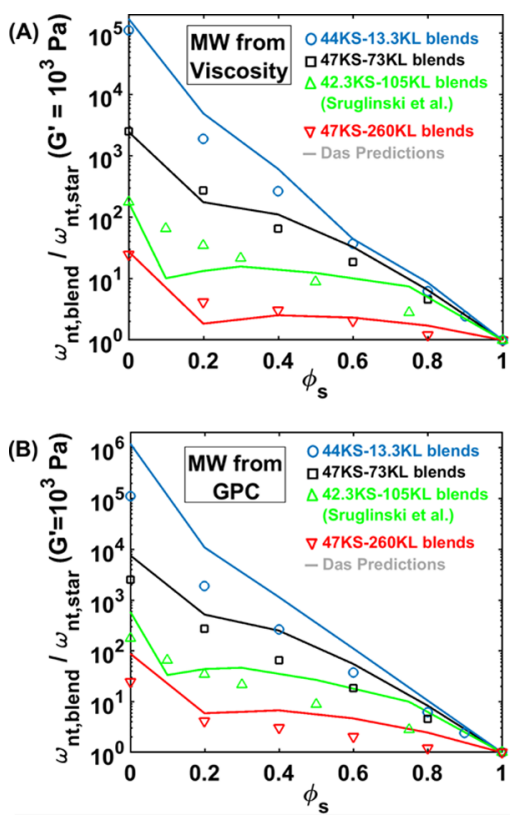

Figure 9. Same as Figure 8 but for the star-linear blends of 44KS$13.3 \mathrm{KL}, 47 \mathrm{KS}-73 \mathrm{KL}$, and $42.3 \mathrm{KS}-105 \mathrm{KL}$ taken from Struglinski et al. $^{33}$ and $47 \mathrm{KS}-260 \mathrm{KL}$.

underpredicts the experimental data by factors of 2.6 and 2.3, respectively. For the $47 \mathrm{KS}-73 \mathrm{KL}$ blend series, predictions differ from data by no more than a factor of 1.8 for any blend.

In contrast to the model agreements for the cases of $44 \mathrm{KS}-$ $13.3 \mathrm{KL}$ and $47 \mathrm{KS}-73 \mathrm{KL}$ blends, the model fails more seriously to predict the relaxation of the $42.3 \mathrm{KS}-105 \mathrm{KL}$ blends, from Struglinski et al. ${ }^{33}$ in Figure 9A. Not only is there a deviation of up to a factor 6.4 for the $42.3 \mathrm{KS}-105 \mathrm{KL}\left(\phi_{\mathrm{s}}=\right.$ 0.1 ) blend, but also the model inaccurately predicts a nonmonotonic dependence on $\phi_{s}$, while the experimental data show roughly a linear dependence. In addition, the hierarchical model incorrectly predicts non-monotonicity in the $47 \mathrm{KS}-$ $260 \mathrm{KL}$ blends; however, in this case, the predicted terminal frequency for the $\phi_{\mathrm{s}}=0.2$ blend is only a factor 1.4 lower than that for the $\phi_{\mathrm{s}}=0.4$ blend. This modest predicted nonmonotonicity is absent from the experimental rheology, for which the near-terminal frequency for the $\phi_{\mathrm{s}}=0.2$ blend is a factor of 1.4 higher than that for the $\phi_{\mathrm{s}}=0.4$ blend.

The model predictions that utilize the pure star and pure linear molecular weights from GPC, as shown in Figure 9B, obtain similar success in superposing the experimental data as in Figure 9A. Although the terminal frequencies of the pure linear polymers are captured rather poorly in comparison to Figure 9A, the predictions in Figure 9B lead to the same conclusions as those of Figure 9A. We note that model predictions of the $44 \mathrm{KS}-13.3 \mathrm{KL}$ blends in Figure 9B may appear considerably poorer than those of Figure 9A; however, this discrepancy is due to the model's inability to capture accurately the relaxation of both the pure $44 \mathrm{KS}$ and the pure $13.3 \mathrm{KL}$ samples when using the molecular weights given by GPC, as shown in Figure S10 of the Supporting Information. Despite this shortcoming, Figure S10 shows that model predictions of the resulting $44 \mathrm{KS}-13.3 \mathrm{KL}$ blends superpose reasonably well with the experimental data.

We next compare in Figures 10-14 model predictions of $G^{\prime}$ with the corresponding experimental data for multiple blend series. The $G^{\prime \prime}$ data for these star-linear blends are presented 

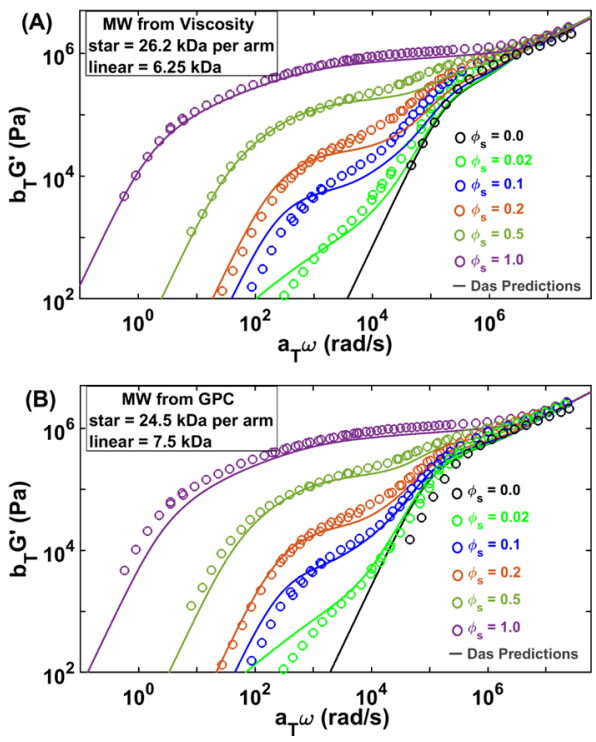

Figure 10. Experimental (symbols) $G^{\prime}$ linear rheology data of the $25.4 \mathrm{KS}-7.5 \mathrm{KL}$ blend series, obtained from Shivokhin et al., ${ }^{34}$ for star volume fractions $\left(\phi_{\mathrm{s}}\right) 0,0.02,0.1,0.2,0.5$, and 1 , compared with predictions of the hierarchical model (A) using zero-shear viscositydefined molecular weights and (B) using GPC-measured molecular weights. As described elsewhere in this section, the molecular weights used in the predictions are given in the legend.
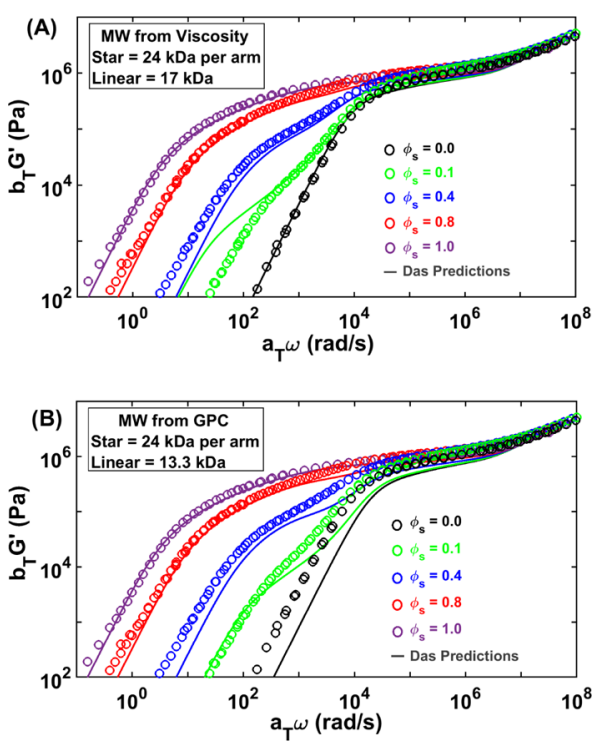

Figure 11. Same as Figure 10, but for the 24KS-13.3KL blend series.

in the Supporting Information. Figure $10 \mathrm{~A}$ compares the rheology of the $24.5 \mathrm{KS}-7.5 \mathrm{KL}$ blend series (symbols) taken from Shivokhin et al. ${ }^{34}$ against the hierarchical model predictions (lines) with molecular weights adjusted to fit the pure materials as listed in Table 5, while Figure 10B shows the same comparison, but with model predictions using molecular weights defined by GPC. Model predictions superpose reasonably well with the experimental data but with deviations in Figure $10 \mathrm{~A}$ at star volume fractions of $\phi_{\mathrm{s}}=0.5,0.2$, and 0.1 , especially at frequencies just above the terminal range. Figure 10B displays similar results, despite the model underpredicting slightly the pure $24.5 \mathrm{KS}$ sample and overpredicting slightly the pure $7.5 \mathrm{KL}$ sample. We note that a modified version of the time-marching algorithm (TMA) was successful in predicting
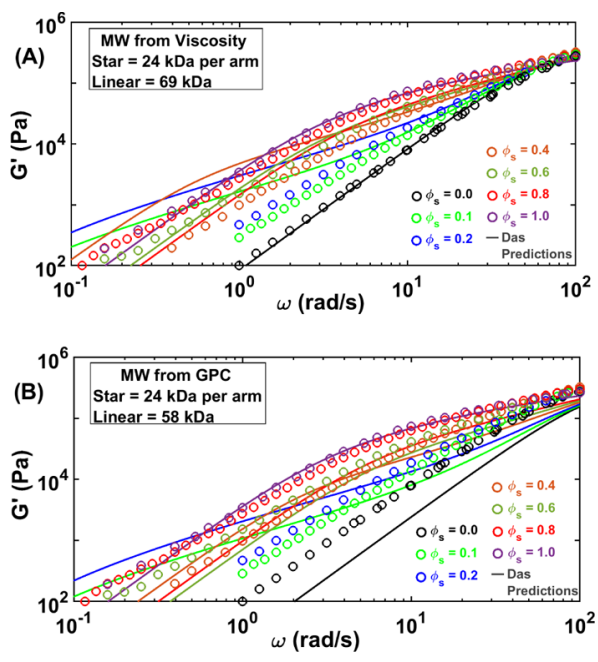

Figure 12. Same as Figure 10 but for the $24 \mathrm{KS}-58 \mathrm{KL}$ blend series. Experimental data was taken from Desai et al. ${ }^{32}$
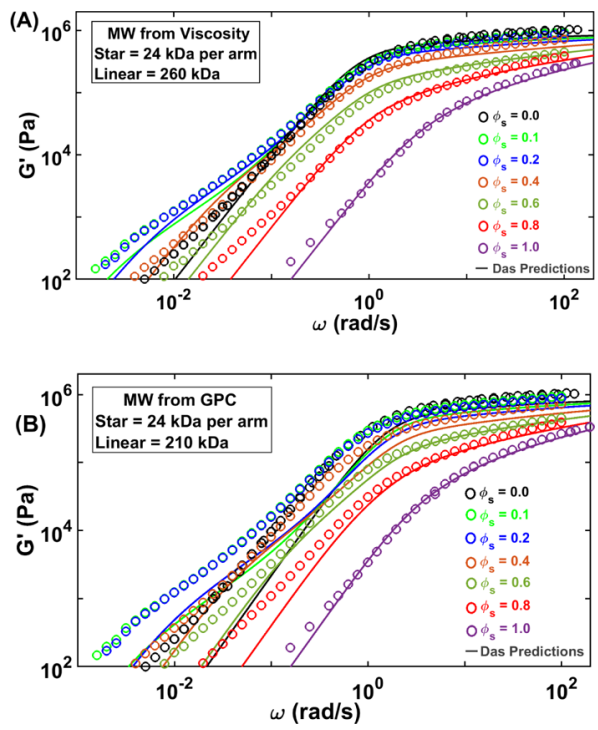

Figure 13. Same as Figure 10 but for the 24KS-210KL blend series.
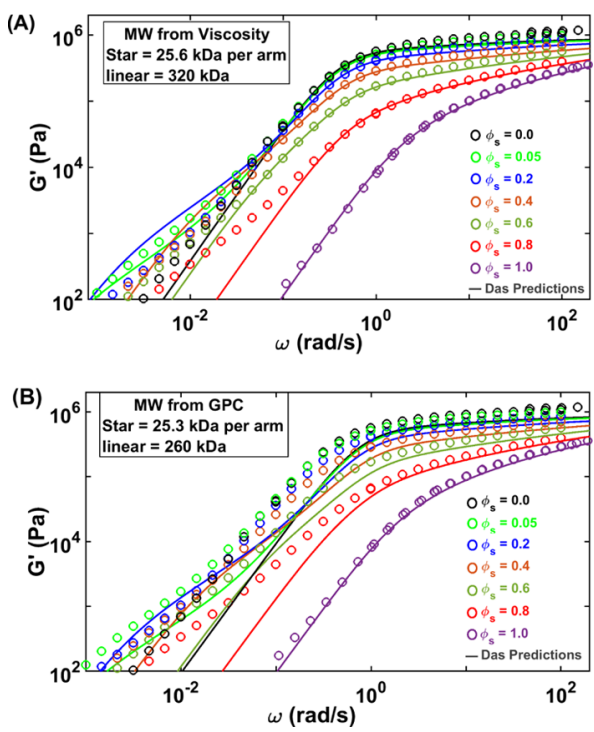

Figure 14. Same as Figure 10 but for the 25.3KS-260KL blend series. 
the $24.5 \mathrm{KS}-7.5 \mathrm{KL}$ blends, as reported by Ebrahimi et al. ${ }^{65}$ This particular TMA model employs a self-consistent definition of CR-Rouse relaxation that assumes that the slowly relaxing star arms diffuse in the fat tube at a rate that is set by the motion of the linear chains.

Figure 11 is similar to Figure 10, except for the 24KS13.3KL blend series. Despite adjusting molecular weights to match the pure star and the pure linear relaxation, as well as successful modeling of low-frequency crossover frequency $\omega_{x, t}$ in Figure 11A, the terminal relaxation is incorrectly predicted for low star volume fractions, especially for $\phi_{\mathrm{s}}=0.1$. In contrast, Figure 11B shows predictions for the $\phi_{\mathrm{s}}=0.1$ blend that agree with the data when the GPC molecular weights are assumed; however, agreement for the $\phi_{\mathrm{s}}=0.1$ blend is likely a consequence of the model's underprediction for the pure 13.3KL sample.

In the experiments of Figure 11, the lifetimes of the relatively dilute, long-lived star-star entanglements seem to be reduced by the linear chains, resulting in faster CR-Rouse relaxation than predicted by the tube model. This accelerated relaxation might be due to additional relaxation mechanisms, such as the thin tube contour-length fluctuations discussed by Read et al. ${ }^{66}$ or the "tension re-equilibration" mechanism discussed by van Ruymbeke. ${ }^{40,41}$ Alternatively, and possibly in conjunction with the failure of CR-Rouse physics, the tube model may not be capturing correctly the dynamic dilution physics in the limit of sparse star-star entanglement interactions present in star-linear blends of high linear content. Note that the difference in terminal relaxation between the pure star and the pure linear samples for this $24 \mathrm{KS}-13.3 \mathrm{KL}$ blend series is roughly 3 orders of magnitude, as opposed to the 4-plus orders of magnitude difference between the pure $24.5 \mathrm{KS}$ and the pure $7.5 \mathrm{KL}$ melts in Figure 10. As remarked earlier, the tube model seems to fail increasingly as the ratio of the relaxation time of the linear polymer to that of the star increases toward unity.

Next, we revisit in Figure 12 the comparison of hierarchical model predictions (lines), again with the Das parameters and thin tube assumption, for the $25 \mathrm{KS}-58 \mathrm{KL}$ blends (symbols), initially reported by Desai et al. ${ }^{32}$ As in Figures 10A and 11A, and unlike the modeling in Desai et al., the molecular weights were adjusted to fit the rheology of the pure $24 \mathrm{KS}$ and the pure $58 \mathrm{KL}$ samples in Figure 12A. Despite this adjustment, and consistent with the original findings reported in Desai et al., model predictions fail to match the blend data, with the exception of the $24 \mathrm{KS}-58 \mathrm{KL}\left(\phi_{\mathrm{s}}=0.6\right)$ blend. Furthermore, the model predictions in Figure 12A falsely predict nonmonotonicity, with terminal relaxation times for star volume fractions $\phi_{\mathrm{s}}=0.1,0.2$, and 0.4 exceeding those for both the pure star and the pure linear polymers. Similar model failure is observed in Figure 12B, when the GPC molecular weights are used in model predictions; however, we note that using the GPC molecular weights in the model leads to notable underprediction of the pure 58KL experimental data. Finally, we note that the relaxation time of the pure star is less than an order of magnitude different from that of the linear sample, regardless of how the molecular weight is defined. As we have noted, the increasing ratio of linear to star relaxation times is associated with an increasing disagreement between the data and the tube model.

In Figures 13 and 14, we report results for star-linear blends in which the pure linear component has a longer relaxation time than the pure star component. The experimental data of the $24 \mathrm{KS}-210 \mathrm{KL}$ blends in Figure 13, with star volume fractions $\phi_{\mathrm{s}}=0.4,0.2$, and 0.1 , have longer relaxation times than both the pure linear and pure star components. We note that the hierarchical model predicts this non-monotonic relaxation of the star-linear blends, regardless of whether molecular weights in model predictions are defined by GPC or the zero-shear viscosity fits shown in Table 5. However, we note that the zero-shear viscosity molecular weights yield better model agreement with the experimental data in Figure 13A than do the GPC molecular weights in Figure 13B.

We note in Figure 13 that the terminal rheology of the pure $210 \mathrm{KL}$ experimental data shows a slight tail at low frequency, indicating the presence of some higher-molecular-weight species within the melt. While this tail is small, we investigated the possibility that it might be responsible for the observed non-monotonicity in the Supporting Information by using the hierarchical model to determine the molecular weight and volume fraction of long linear chains needed in the pure linear material to fit the pure linear data and then seeing the effect of this on the $24 \mathrm{KS}-210 \mathrm{KL}$ blend predictions. Despite having fit the polydispersity-induced long tail of the pure $210 \mathrm{KL}$ sample, model predictions for the blend are only slightly changed and continue to show non-monotonicity in the dependence of terminal relaxation time on star concentration. While it is always possible that this non-monotonicity might conceivably be absent in rigorously monodisperse materials, it remains the case that, whatever causes it, we find a non-monotonic dependence of terminal relaxation time on the blending ratio of a linear, slightly polydisperse, sample, with a star polymer.

The experimentally observed non-monotonicity can perhaps be explained by the extreme sensitivity of star polymers to constraint release. Consider a pure star polymer that in the absence of constraint release would relax slower than the linear polymer but in the presence of constraint release by dynamic dilution relaxes faster than the linear polymer. Since the dynamic dilution of the pure star comes from fast relaxation of the tips of the star arms, the presence of a majority fraction of long linear polymers greatly suppresses this dilution and greatly slows the relaxation of the star, enough to push its terminal time much closer to that of the star without constraint release and slower than the linear polymer itself relaxes. If the star is the minority component, the fast relaxation of the tips of the arm has little effect on the relaxation of the linear chain. Hence, the linear chain acts as a strong suppressor of dynamic dilution of the star arm, enough that the star terminal relaxation time reverts to a value closer to that of a star with no dynamic dilution, which is longer than that of both the pure star with dynamic dilution and of the linear polymer. The work of Matsumiya et $\mathrm{al}^{22}$ showed experimentally that constraint release of the star can in fact be quenched if the star polymer is blended with a very high molecular weight linear polymer at a low star volume fraction; in this case, the star relaxation time is enormously slowed. The case of the $24 \mathrm{KS}-210 \mathrm{KL}$ blend in Figure 13 is a less extreme example of the star-polymer system in Matsumiya et al., where the pure $210 \mathrm{KL}$ polymer has a longer relaxation time, but not by many orders of magnitude larger, than that of the pure $24.5 \mathrm{KS}$ polymer. The $24.5 \mathrm{KS}-$ $210 \mathrm{KL} \phi_{\mathrm{s}}=0.2$ and 0.1 blends likely recreate the constraint release limitations on the star component, to some extent similar to that in Matsumiya et al. If this mechanism of nonmonotonic dependence of terminal relaxation rate on blend composition is correct, then the tube model correctly captures the effect for long enough linear chains but greatly overpredicts 
its importance for linear molecules of more modest length. This insight perhaps provides a starting point for improving the tube model so that its predictions match experimental data for a wider range of blends.

To further confirm this non-monotonic behavior, we acquired new star and linear 1,4-polybutadiene samples, similar in molecular weights to those of Figure 13. The new linear component, $260 \mathrm{KL}$, was synthesized and characterized by our team (Hadjichristidis lab), while the linear polymer in Figure $13,210 \mathrm{KL}$, was purchased from Polymer Source, thus giving us similar material from two different labs. In Figure 14A, the linear rheology of the $25.3 \mathrm{KS}-260 \mathrm{KL}$ blends again displays non-monotonic behavior, again in agreement with predictions of the hierarchical model when the zero-shear-viscosity molecular weights are assumed. The model also predicts non-monotonicity when the GPC molecular weights are used, as shown in Figure 14B; however, these predictions in Figure $14 \mathrm{~B}$ agree less well with the experimental data than do those in Figure 14A. Slight polydispersity is also evident in the terminal rheology of the pure $260 \mathrm{KL}$ experimental data, which we again explore in the Supporting Information and show that it does not change the non-monotonic behavior significantly.

We note that the hierarchical model correctly predicts nonmonotonic behavior in the $24 \mathrm{KS}-210 \mathrm{KL}$ and the $25.3 \mathrm{KS}-$ $260 \mathrm{KL}$ blend series, but it also incorrectly predicts it for the $24 \mathrm{KS}-58 \mathrm{KL}$ blend series depicted in Figure 12. In Figure 12, however, the pure star melt relaxes more slowly than the linear melt, while the reverse is the case in Figures 13 and 14. It is noteworthy that the CFSM slip-link model of Schieber and coworkers correctly predicts monotonic behavior of the terminal relaxation time with blend composition for the $24 \mathrm{KS}-58 \mathrm{KL}$ blend data. ${ }^{32}$ It would be of great interest to see whether sliplink models predict non-monotonic behavior of blends in which the linear melts relax slower than the star ones. If not, this would represent a case in which the tube model captures constraint release behavior that is not captured by slip-link models. This would indicate a subtlety in constraint-release dynamics that is not yet consistently implemented in either class of models. A careful study of the source of the nonmonotonicity in the tube model and its lack in slip-link models might then open the door to deeper understanding of constraint release in general and to improvements in the modeling of constraint release in tube and slip-link models. We believe that since constraint release is so difficult to model consistently in star-linear blends, a future model that successfully predicts the rheology of these blends is likely to be successful also for polydisperse mixtures of well-entangled star and linear blends. This, in turn, would provide a strong basis for consistently accurate modeling of commercial branched polymer melts.

Finally, to summarize our results, in Figure 15, we organize our data onto a "phase map" of the zero-shear viscosity $\left(\eta_{\mathrm{o}}\right)$, obtained from the experiment, of the pure star paired with that of its pure linear counterpart, labeling each of the 10 blend series with a symbol of its own distinct color. Along the red dashed line, the zero-shear viscosities of the pure star and linear polymers are equal. To the right of the blue line in Figure 15, the hierarchical model predicts non-monotonicity, while to the left, it does not. The model falsely predicts nonmonotonicity in all of the blends displayed to the right of the blue solid line, but to the left of the red dashed line, namely, the $24 \mathrm{KS}-13.3 \mathrm{KL}, 24 \mathrm{KS}-58 \mathrm{KL}, 25.3 \mathrm{KS}-73 \mathrm{KL} * *$, $47 \mathrm{KS}-$ $73 \mathrm{KL}, 42.3 \mathrm{KS}-105 \mathrm{KL}$, and $47 \mathrm{KS}-260 \mathrm{KL}$ blends. The

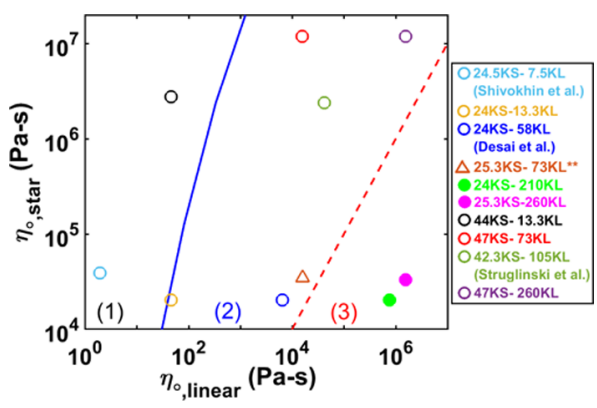

Figure 15. Experimental zero-shear viscosities of each pure star plotted against those of the linear polymer for each of the 10 blend series (symbols). The blue line indicates the transition from monotonic (left of the line) to non-monotonic (right of the line) dependence of terminal relaxation on composition in hierarchical model predictions. The dashed red line marks equality in the zeroshear viscosities of the pure linear and the pure star melts. The filled symbols represent the two blend series for which both theory and experiment show non-monotonic dependence of the terminal relaxation time on blend composition. We note that the $25.3 \mathrm{KS}-$ $73 \mathrm{KL}$ blend was prepared and tested at the end of our study to confirm the absence of monotonicity in the experimental blend data when the zero-shear viscosities of pure $25.3 \mathrm{KS}$ and pure $73 \mathrm{KL}$ blends are close to, but to the left of, the red dashed line.

$25.3 \mathrm{KS}-73 \mathrm{KL}$ blend was prepared and tested, after completion and plotting of the others, to further verify the absence of non-monotonicity in the experimental data, although predicted to be present by the hierarchical model, when the zero-shear viscosities of the pure star and pure linear polymers are near, but to the left of the red dashed line. To the right of the red dashed line, the non-monotonic predictions prove accurate for the $24 \mathrm{KS}-210 \mathrm{KL}$ and the $25.3 \mathrm{KS}-260 \mathrm{KL}$ blends.

We note that Figure 15 places the $24 \mathrm{KS}-13.3 \mathrm{KL}$ and the $47 \mathrm{KS}-73 \mathrm{KL}$ series in the region for which non-monotonicity is predicted by the theory. While this might seem to conflict with the apparently monotonic dependence of terminal relaxation time on $\phi_{s}$ seen, respectively, in Figures 11 and S6 (the latter in the Supporting Information), the presence of non-monotonicity is revealed in Figures 16 and 17 when we include model predictions (pink lines) of additional 24KS$13.3 \mathrm{KL}$ and $47 \mathrm{KS}-73 \mathrm{KL}$ blend compositions that were not explored experimentally within the existing data sets (pink symbols). With the addition of the $24 \mathrm{KS}-13.3 \mathrm{KL} \phi_{\mathrm{s}}=0.2$ and the $47 \mathrm{KS}-73 \mathrm{KL} \phi_{\mathrm{s}}=0.1$ blends, respectively, to Figures 16 and 17 , we observe clearly the emergence of non-monotonicity in the predictions for both of these blend series, yet the experimental data retain a monotonic dependence of terminal relaxation on $\phi_{s}$. Non-monotonicity is falsely predicted whether the molecular weights used in the predictions are obtained from zero-shear viscosity fitting or from GPC. This confirms the accuracy of the boundary given by the blue line in Figure 15 separating the predictions of non-monotonicity (to the right of the solid blue line) from the predictions of monotonic composition dependence (to the left of the solid blue line).

\section{CONCLUSIONS}

The limits of an advanced tube model, the hierarchical model with the "standard" Das parameter set and the assumption of primitive path fluctuations in the thin tube during constraintrelease Rouse (CR-Rouse) relaxation, were thoroughly tested against the linear rheology of 10 series of 1,4-polybutadiene 

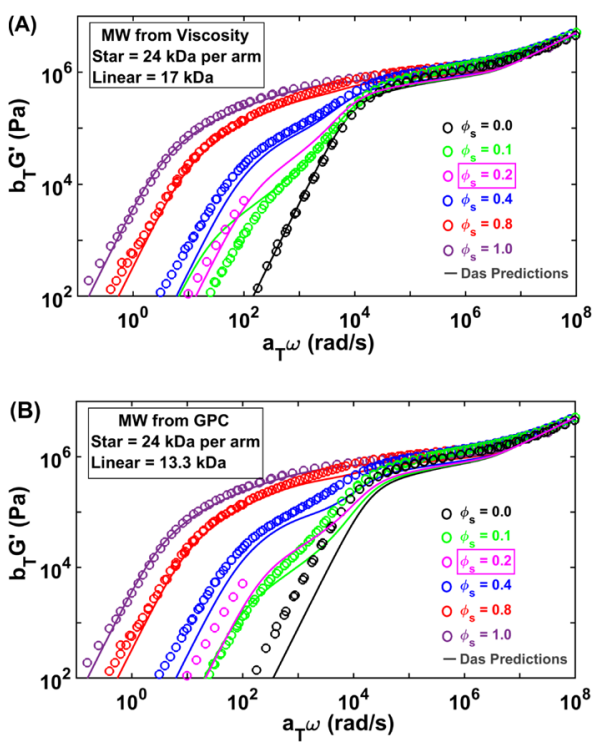

Figure 16. Experimental (symbols) linear rheology data of the 24KS13.3KL blend series for star volume fractions $\left(\phi_{\mathrm{s}}\right) 0,0.1, * 0.2 *, 0.4$, 0.8 , and 1 , compared with predictions of the hierarchical model that use star and linear molecular weights obtained from (A) zero-shear viscosity and (B) GPC. The $24 \mathrm{KS}-13.3 \mathrm{KL}\left(\phi_{\mathrm{s}}=0.2\right)$ blend (pink symbols) was prepared after the others to confirm the inaccuracy of the prediction of non-monotonic dependence of terminal relaxation in $G^{\prime}$ on composition at low star volume fraction.
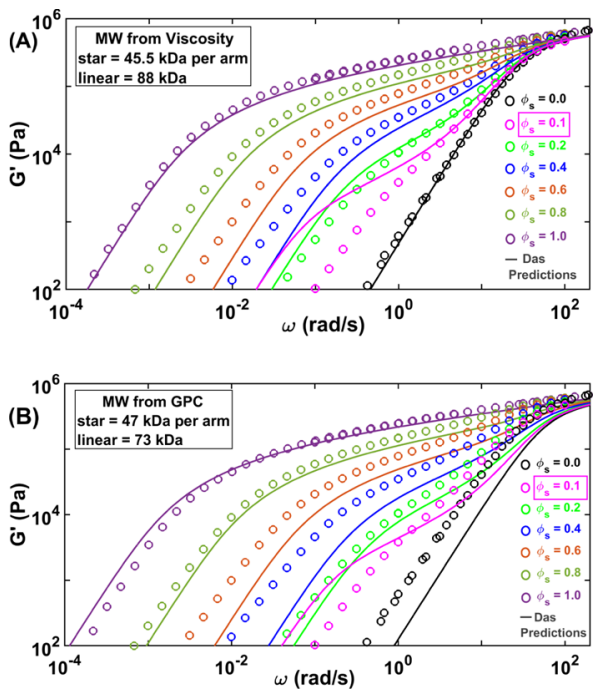

Figure 17. As in Figure 16, the 47KS-73KL $\left(\phi_{\mathrm{s}}=0.1\right)$ was prepared after the others to confirm the absence of non-monotonicity in the experiments.

star-linear blends at several volume fractions of stars. Seven of these series were produced using three newly synthesized stars, which were mixed with linear polymers either synthesized or purchased. The 1,2-vinyl contents of the new pure star and pure linear melts were found to be within the range $5-10 \%$ by ${ }^{1} \mathrm{H}$ NMR and by comparing the WLF horizontal shift factors with literature references. The accuracy of GPC and TGIC characterization of the molecular weights of our new materials was assessed by comparing their zero-shear viscosities with the molecular-weight dependencies of viscosity drawn from data in the literature for 1,4-polybutadiene star and linear polymers, and molecular weights were found generally to be within around $20 \%$ of each other. We then compared predictions of the hierarchical model against the linear rheology of 10 series of star-linear blends, 7 of which were newly prepared samples and the other 3 were drawn from the existing literature, for a total over 50 samples, providing the most comprehensive data base of star-linear blends ever assembled for any polymer chemistry.

To be sure that our results are robust to possible modest errors in sample characterization and in the tube model as applied to the pure materials, we assessed the hierarchical model using both the GPC-characterized molecular weights of the pure star and linear melts and the molecular weights needed to gain agreement of the model with the measured zero-shear viscosities of all pure components. Using either method, reasonably good agreement of measured and predicted linear viscoelasticity of the star-linear blends is obtained when the terminal relaxation time of the linear polymer is more than 3 orders of magnitude shorter than that of the star polymer. Agreement worsens markedly as the terminal relaxation time of the linear polymer approaches more closely that of the star, with the hierarchical model incorrectly predicting a "non-monotonic" behavior in which the terminal relaxation time of some of the blends is longer than that of either the pure star or pure linear polymer in the blend. Remarkably, once the terminal relaxation time of the linear polymer becomes longer than that of the star, the experimental results actually confirm this predicted non-monotonic behavior, and the frequency-dependent moduli are in good agreement with the hierarchical model. Thus, our thorough study of these many samples reveals surprising successes and surprising failures of the tube model. The most surprising success is the prediction of the non-monotonic dependence of terminal relaxation time on blend composition when the pure linear chain relaxes more slowly than does the pure star chain. The most surprising failure, remarkably enough, is that the model continues to predict this non-monotonic behavior even when the pure linear melt relaxes more rapidly, but not too much more rapidly than the pure star melt, while the experimental data revert to a monotonic dependence on the star volume fraction in these cases. These successes and failures are robust, as both were revealed in more than one blend series. Our previous very detailed study on three of the same star-linear blend series showed that variations of the tube model, obtained by using the BoB formulation, by deviating from the thin tube assumption, by allowing disentanglement relaxation, or by changing tube model parameters, fail to bring the tube model into even approximate agreement with more than a fraction of the relaxation data. Thus, the new work reported here, combined with our previous study, demonstrates that, despite remarkably accurate predictions in some cases, no widely used version of the tube model is able to predict rheology consistently for all entangled star-linear blends with the same chemical structure.

The study thus demonstrates the remarkably subtle effects of constraint release in star-linear blends and the ability of an advanced version of the tube model to capture such phenomena for some blends, although not accurately enough to predict the range of molecular weights and compositions over which they occur. The work presented here, while not overcoming the limitations of the tube model, does help define the conditions under which it succeeds or fails and thus suggests directions for future research. Our work also provides extensive data sets that can be used for testing other tube 
models, slip-link models, or other theories and simulations that might be forthcoming.

\section{ASSOCIATED CONTENT}

\section{S Supporting Information}

The Supporting Information is available free of charge on the ACS Publications website at DOI: 10.1021/acs.macromol.9b00642.

Details concerning the synthesis and characterization of the $73 \mathrm{KL}, 260 \mathrm{KL}, 25.3 \mathrm{KS}, 44 \mathrm{KS}$, and $44 \mathrm{KS} 1,4-$ polybutadiene melts; characterization of the $13.3 \mathrm{KL}$ 1,4-polybutadiene purchased from Polymer Source containing additional rheology and analysis of both freshly prepared 1,4-polybutadiene star-linear blends and select star-linear blends, of the same chemistry, borrowed from the literature (PDF)

\section{AUTHOR INFORMATION}

\section{Corresponding Authors}

*E-mail: Nikolaos.Hadjichristidis@kaust.edu.sa (N.H.).

*E-mail: rlarson@umich.edu (R.G.L.).

\section{ORCID}

Ryan Hall: 0000-0002-5567-4016

Sanghoon Lee: 0000-0002-8367-1787

Taihyun Chang: 0000-0003-2623-1803

Konstantinos Ntetsikas: 0000-0002-9236-931X

George Polymeropoulos: 0000-0002-3352-0948

Nikos Hadjichristidis: 0000-0003-1442-1714

Ronald G. Larson: 0000-0001-7465-1963

\section{Present Addresses}

ODepartment of Chemical and Materials Engineering, New Jersey Institute of Technology, Newark, New Jersey 07102, United States (D.C.V.).

${ }^{\nabla}$ LG Chem, Ltd., LG Science Park, Seoul 07796, Republic of Korea (B.-G.K.).

\section{Notes}

The authors declare no competing financial interest.

Deceased (Q.H.).

\section{ACKNOWLEDGMENTS}

R.H. and R.G.L. gratefully acknowledge the support of the National Science Foundation, under grants DMR 1403335 and 1707640. Any opinions, findings, and conclusions or recommendations expressed in this material are those of the authors and do not necessarily reflect the views of the National Science Foundation (NSF). N.H. gratefully acknowledges the support of the King Abdullah University of Science and Technology (KAUST).

\section{REFERENCES}

(1) Doi, M.; Edwards, S. F. Dynamics of Concentrated Polymer Systems Part 1: Brownian Motion in the Equilibrium State. J. Chem. Soc., Faraday Trans. 2 1978, 74, 1789-1801.

(2) Doi, M.; Edwards, S. F. Dynamics of Concentrated Polymer Systems Part 2: Molecular Motion Under Flow. J. Chem. Soc., Faraday Trans. 2 1978, 74, 1802-1817.

(3) Doi, M.; Edwards, S. F. Dynamics of Concentrated Polymer Systems Part 3: The Constitutive Equation. J. Chem. Soc., Faraday Trans. 2 1978, 74, 1818-1832.

(4) Doi, M.; Edwards, S. F. Dynamics of Concentrated Polymer Systems Part 4: Rheological Properties. J. Chem. Soc., Faraday Trans. 2 1979, 75, 38-54.
(5) de Gennes, P. G. Reptation of a Polymer Chain in the Presence of Fixed Obstacles. J. Chem. Phys. 1971, 55, 572.

(6) Rubinstein, M. Discretized Model of Entangled-Polymer Dynamics. Phys. Rev. Lett. 1987, 59, 1946-1949.

(7) O'Connor, N. P. T.; Ball, R. C. Confirmation of the Doi Edwards Model. Macromolecules 1992, 25, 5677-5682.

(8) de Gennes, P. G. Dynamics of Entangled Polymer Solutions. II. Inclusion of Hydrodynamic Interactions. Macromolecules 1976, 9, 594-598.

(9) de Gennes, P. G. Theory of Polymer Absorption. J. Phys. 1976, $37,1445-1452$.

(10) Doi, M.; Graessley, W. W.; Helfand, E.; Pearson, D. S. Dynamics of Polymers in Polydisperse Melts. Macromolecules 1987, 20, 1900-1906.

(11) Viovy, J.; Rubinstein, M.; Colby, R. Constraint Release in Polymer Melts: Tube Reorganization versus Tube Dilation. Macromolecules 1991, 24, 3587-3596.

(12) Marrucci, G. Relaxation by Reptation and Tube Enlargement: A Model for Polydisperse Polymers. J. Polym. Sci., Polym. Phys. Ed. 1985, 23, 159-177.

(13) Klein, J. The Onset of Entangled Behavior in Semidilute and Concentrated Polymer Solutions. Macromolecules 1978, 11, 852-858.

(14) Daoud, M.; de Gennes, P. G. Some Remarks on the Dynamics of Polymer Melts. J. Polym. Sci., Polym. Phys. Ed. 1979, 17, 19711981

(15) Ball, R. C.; McLeish, T. C. B. Dynamic Dilution and the Viscosity of Star-Polymer Melts. Macromolecules 1989, 22, 19111913.

(16) Milner, S. T.; McLeish, T. C. B. Parameter-Free Theory for Stress Relaxation in Star Polymer Melts. Macromolecules 1997, 30, 2159-2166

(17) Milner, S. T.; McLeish, T. C. B.; Young, R. N.; Hakiki, A.; Johnson, J. M. Dynamic Dilution, Constraint-Release, and Star-Linear Blends. Macromolecules 1998, 31, 9345-9353.

(18) Watanabe, H.; Matsumiya, Y.; Inoue, T. Dielectric and Viscoelastic Relaxation of Highly Entangled Star Polyisoprene: Quantitative Test of Tube Dilation Model. Macromolecules 2002, $35,2339-2357$

(19) Watanabe, H.; Ishida, S.; Matsumiya, Y.; Inoue, T. Test of Full and Partial Tube Dilation Pictures in Entangled Blends of Linear Polyisoprenes. Macromolecules 2004, 37, 6619-6631.

(20) Watanabe, H.; Ishida, S.; Mastsumiya, Y.; Inoue, T. Viscoelastic and Dielectric Behavior of Entangled Blends of Linear Polyisoprenes Having Widely Separated Molecular Weights: Test of Tube Dilation Picture. Macromolecules 2004, 37, 1937-1951.

(21) Matsumiya, Y.; Kumazawa, K.; Masahiro, N.; Urakawa, O.; Watanabe, H. Dielectric Relaxation of Monodisperse Linear Polyisoprene: Contribution of Constraint Release. Macromolecules 2013, 46, 6067-6080.

(22) Matsumiya, Y.; Masubuchi, Y.; Inoue, T.; Urakawa, O.; Liu, C.; van Ruymbeke, E.; Watanabe, H. Dielectric and Viscoelastic Behavior of Star-Branched Polyisoprene: Two Coarse-Grained Length Scales in Dynamic Tube Dilation. Macromolecules 2014, 47, 7637-7652.

(23) Park, S. J.; Shanbhag, S.; Larson, R. G. A Hierarchical Algorithm for Predicting the Linear Viscoelastic Properties of Polymer Melts with Long-Chain Branching. Rheol. Acta 2005, 44, 319-330.

(24) Wang, Z.; Chen, X.; Larson, R. G. Comparing Tube Models for Predicting the Linear Rheology of Branched Polymer Melts. J. Rheol. 2010, 54, 223-260.

(25) Das, C.; Inkson, N. J.; Read, D. J.; Kelmanson, M. A.; McLeish, T. C. B. Computational Linear Rheology of General Branch-onBranch Polymers. J. Rheol. 2006, 50, 207-234.

(26) van Ruymbeke, E.; Keunings, R.; Bailly, C. Prediction of Linear Viscoelastic Properties for Polydisperse Mixtures of Entangled Star and Linear Polymers: Modified Tube-Based Model and Comparison with Experimental Results. J. Non-Newtonian Fluid Mech. 2005, 128, $7-22$. 
(27) van Ruymbeke, E.; Bailly, C.; Keunings, R.; Vlassopoulos, D. A General Methodology to Predict the Linear Rheology of Branched Polymers. Macromolecules 2006, 39, 6248-6259.

(28) Larson, R. G. Combinatorial Rheology of Branched Polymer Melts. Macromolecules 2001, 34, 4556-4571.

(29) Ahmadi, M.; Bailly, C.; Keunings, R.; Nekoomanesh, M.; Arabi, H.; van Ruymbeke, E. Time Marching Algorithm for predicting the Linear Rheology of Monodisperse Comb Polymer Melts. Macromolecules 2011, 44, 647-659.

(30) Park, S. J.; Larson, R. G. Tube Dilation and Reptation in Binary Blends of Monodisperse Linear Polymers. Macromolecules 2004, 37, 597-604.

(31) Park, S. J.; Larson, R. G. Modeling the Linear Viscoelastic Properties of Metallocene-Catalyzed High Density Polyethylenes with Long-Chain Branching. J. Rheol. 2005, 49, 523-536.

(32) Desai, P. S.; Kang, B. G.; Katzarova, M.; Hall, R.; Huang, Q.; Lee, S.; Shivokhin, M.; Chang, T.; Venerus, D. C.; Mays, J.; Schieber, J. D.; Larson, R. G. Challenging Tube and Slip-Link Models: Predicting the Linear Rheology of Blends of Well-Characterized Sar and Linear 1,4-Polybutadienes. Macromolecules 2016, 49, 4964-4977.

(33) Struglinski, M. J.; Graessley, W. W.; Fetters, L. J. Effects of Polydispersity on the Linear Viscoelastic Properties of Entangled Polymers. 3. Experimental Observations on Binary Mixtures of Linear and Star Polybutadienes. Macromolecules 1988, 21, 783-789.

(34) Shivokhin, M. E.; van Ruymbeke, E.; Bailly, C.; Kouloumasis, D.; Hadjichristidis, N.; Likhtman, A. E. Understanding Constraint Release in Star/Linear Polymer Blends. Macromolecules 2014, 47, $2451-2463$

(35) Schieber, J. D.; Andreev, M. Entangled Polymer Dynamics in Equilibrium and Flow Modeled Through Slip Links. Annu. Rev. Chem. Biomol. Eng. 2014, 5, 367-381.

(36) Khaliullin, R. N.; Schieber, J. D. Self-Consistent Modeling of Constraint Release in a Single-Chain Mean-Field Slip-Link Model. Macromolecules 2009, 42, 7504-7517.

(37) Pilyugina, E.; Andreev, M.; Schieber, J. D. Dielectric Relaxation as an Independent Examination of Relaxation Mechanisms in Entangled Polymers Using the Discrete Slip-Link Model. Macromolecules 2012, 45, 5728-5743.

(38) Andreev, M.; Schieber, J. D. Accessible and Quantitative entangled Polymer Rheology Predictions, Suitable for Complex Flow Calculations. Macromolecules 2015, 48, 1606-1613.

(39) Andreev, M.; Feng, H.; Yang, L.; Schieber, J. D. Universality and Speedup in Equilibrium and Nonlinear Rheology Predictions of the Fixed Slip-Link Model. J. Rheol. 2014, 58, 723.

(40) van Ruymbeke, E.; Masubuchi, Y.; Watanabe, H. Effective Value of the Dynamic Dilution Exponent in Bidisperse Linear Polymers: From 1 to 4/3. Macromolecules 2012, 45, 2085-2098.

(41) van Ruymbeke, E.; Shchetnikava, V.; Matsumiya, Y.; Watanabe, H. Dynamic Dilution Effect in Binary Blends of Linear Polymers with Well-Separated Molecular Weights. Macromolecules 2014, 47, 76537665 .

(42) Shahid, T.; Huang, Q.; Oosterlinck, F.; Clasen, C.; van Ruymbeke, E. Dynamic Dilution Exponent in Monodisperse Entangled Polymer Solutions. Soft Matter 2017, 13, 269-282.

(43) Huang, Q.; Hengeller, L.; Alvarez, N. J.; Hassager, O. Bridging the Gap between Polymer Melts and Solutions in Extensional Rheology. Macromolecules 2015, 48, 4158-4163.

(44) Hall, R.; Kang, B.; Lee, S.; Chang, T.; Venerus, D. C.; Hadjichristidis, N.; Mays, J.; Larson, R. G. Determining the Dilution Exponent for Entangled 1,4-Polybutadienes Using Blends of NearMonodisperse Star with Unentangled, Low Molecular Weight Linear Polymers. Macromolecules 2019, 52, 1757-1771.

(45) Pearson, D. S.; Helfand, E. Viscoelastic Properties of StarShaped Polymers. Macromolecules 1984, 17, 888-895.

(46) Park, S. J.; Larson, R. G. Dilution Exponent in the Dynamic Dilution Theory for Polymer Melts. J. Rheol. 2003, 47, 199-211.

(47) Daniels, D. R.; McLeish, T. C. B.; Kant, R.; Crosby, B. J.; Young, R. N.; Pryke, A.; Allgaier, J.; Groves, D. J.; Hawkins, R. J.
Linear Rheology of Diluted Linear, Star and Model Long Chain Branched Polymer Melts. Rheol. Acta 2001, 40, 403-415.

(48) Raju, V. R.; Menezes, E. V.; Marin, G.; Graessley, W. W.; Fetters, L. J. Concentration and Molecular Weight Dependence of Viscoelastic Properties in Linear and Star Polymers. Macromolecules 1981, 14, 1668-1676.

(49) Tao, H.; Huang, C.; Lodge, T. P. Correlation Length and Entanglement Spacing in Concentrated Hydrogenated Polybutadiene Solutions. Macromolecules 1999, 32, 1212-1217.

(50) Brochard, F.; de Gennes, P. G. Dynamical Scaling for Polymers in Theta Solvents. Macromolecules 1977, 10, 1157-1161.

(51) Colby, R. H.; Fetters, L. J.; Graessley, W. W. The Melt Viscosity-Molecular Weight Relationship for Linear Polymers. Macromolecules 1987, 20, 2226-2237.

(52) Struglinski, M. J.; Graessley, W. W. Effects of Polydispersity on the Linear Viscoelastic Properties of Entangled Polymers. 1. Experimental Observations for Binary Mixtures of Linear Polybutadiene. Macromolecules 1985, 18, 2630-2643.

(53) Roovers, J. Properties of the Plateau Zone of Star-Branched Polybutadienes and Polystyrenes. Polymer 1985, 26, 1091-1095.

(54) Roovers, J. Tube Renewal in the Relaxation of 4-Arm Star Polybutadienes in Linear Polybutadienes. Macromolecules 1987, 20, $148-152$.

(55) Park, S. J.; Desai, P. S.; Chen, X.; Larson, R. G. Universal Relaxation Behavior of Entangled 1,4-Polybutadiene Melts in the Transition Frequency Region. Macromolecules 2015, 48, 4122-4131.

(56) Li, S. W.; Park, H. E.; Dealy, J. M. Evaluation of Molecular Linear Viscoelastic Models for Polydisperse H Polybutadienes. J. Rheol. 2011, 55, 1341-1373.

(57) Palade, L. I.; Verney, V.; Attane, P. Time-Temperature Superposition and Linear Viscoelasticity of Polybutadienes. Macromolecules 1995, 28, 7051.

(58) Chang, T. Polymer Characterization by Interaction Chromatography. J. Polym. Sci., Part B: Polym. Phys. 2005, 43, 1591.

(59) Perny, S.; Allgaier, J.; Cho, D.; Lee, W.; Chang, T. Synthesis and Structural Analysis of an H-Shaped Polybutadiene. Macromolecules 2001, 34, 5408.

(60) Li, S. W.; Park, H. E.; Dealy, J. M.; Maric, M.; Lee, H.; Im, K.; Choi, H.; Chang, T.; Rahman, M. S.; Mays, J. Detecting Structural Polydispersity in Branched Polybutadienes. Macromolecules 2011, 44, 208.

(61) Chen, X.; Rahman, M. S.; Lee, H.; Mays, J.; Chang, T.; Larson, R. G. Combined Synthesis, TGIC Characterization, and Rheological Measurement and Prediction of Symmetric H Polybutadienes and Their Blends with Linear and Star-Shaped Polybutadienes. Macromolecules 2011, 44, 7799-7809.

(62) Hadjichristidis, N.; Roovers, J. Linear Viscoelastic Properties of Mixtures of 3- and 4-arm Polybutadiene Stars. Polymer 1985, 26, $1087-1090$.

(63) Shanbhag, S.; Larson, R. G.; Takimoto, J.; Doi, M. Deviations from Dynamic Dilution in the Terminal Relaxation of Star Polymers. Phys. Rev. Lett. 2001, 87, No. 195502.

(64) Cao, J.; Wang, Z. Microscopic Picture of Constraint Release Effects in Entangled Star Polymer Melts. Macromolecules 2016, 49, $5677-5691$.

(65) Ebrahimi, T.; Taghipour, H.; Grießl, D.; Mehrhodavandi, P.; Hatzikiriakos, S. G.; van Ruymbeke, E. Binary Blends of Entangled Star and Linear Poly(hydroxybutyrate): Effect of Constraint Release and Dynamic Tube Dilation. Macromolecules 2017, 50, 2535-2546.

(66) Read, D. J.; Jagannathan, K.; Sukumaran, S. K.; Auhl, D. A FullChain Constitutive Model for Bidisperse Blends of Linear Polymers. J. Rheol. 2012, 56, 823-873. 